\title{
Global Dynamics of Some Exponential Type Systems
}

\author{
A. Q. Khan ${ }^{10},{ }^{1}$ H. M. Arshad, ${ }^{1}$ B. A. Younis, ${ }^{2}$ KH. I. Osman, ${ }^{3}$ Tarek F. Ibrahim, ${ }^{4,5}$ \\ I. Ahmed $\mathbb{D}$, ${ }^{6}$ and A. Khaliq $\mathbb{D}^{7}$ \\ ${ }^{1}$ Department of Mathematics, University of Azad Jammu and Kashmir, Muzaffarabad 13100, Pakistan \\ ${ }^{2}$ Department of Mathematics, Faculty of Sciences and Arts in Zahran Alganoob, King Khalid University, Abha, Saudi Arabia \\ ${ }^{3}$ Department of Mathematics, Faculty of Sciences and Arts in Sarat Abeda, King Khalid University, Abha, Saudi Arabia \\ ${ }^{4}$ Department of Mathematics, Faculty of Sciences and Arts in Mahayel Aseer, King Khalid University, Abha, Saudi Arabia \\ ${ }^{5}$ Department of Mathematics, Faculty of Sciences, Mansoura University, Mansoura 35516, Egypt \\ ${ }^{6}$ Department of Mathematics, Mirpur University of Science and Technology (MUST), Mirpur 10250 (AJK), Pakistan \\ ${ }^{7}$ Department of Mathematics, Riphah International University, Lahore Campus, Lahore, Pakistan
}

Correspondence should be addressed to A. Q. Khan; abdulqadeerkhan1@gmail.com

Received 8 January 2020; Revised 23 February 2020; Accepted 7 April 2020; Published 26 May 2020

Guest Editor: Qasem M. Al-Mdallal

Copyright (c) 2020 A. Q. Khan et al. This is an open access article distributed under the Creative Commons Attribution License, which permits unrestricted use, distribution, and reproduction in any medium, provided the original work is properly cited.

We explore the boundedness and persistence, existence of an invariant rectangle, local dynamical properties about the unique positive fixed point, global dynamics by the discrete-time Lyapunov function, and the rate of convergence of some (2,3)-type exponential systems of difference equations. Finally, theoretical results are numerically verified.

\section{Introduction}

Recently, global dynamical properties of $(2,2)$ as well as $(2,3)$-type exponential difference equations or systems of exponential difference equations have widely been explored. In this regard, Ozturk et al. [1] have explored the global dynamical properties of the following $(2,2)$-type exponential difference equation:

$$
x_{n+1}=\frac{\alpha_{10}+\alpha_{11} e^{-x_{n}}}{\alpha_{12}+x_{n-1}},
$$

where $\alpha_{s}(s=10,11,12)$ and $x_{s}(s=-1,0)$ are the positive real numbers. Equation (1) may be viewed as a model in mathematical biology where $\alpha_{10}$ is the immigration rate, $\alpha_{11}$ is the population growth rate, and $\alpha_{12}$ is the carrying capacity. Bozkurt [2] has explored the global dynamical properties of the following $(2,3)$-type exponential difference equation:

$$
x_{n+1}=\frac{\alpha_{10} e^{-x_{n}}+\alpha_{11} e^{-x_{n-1}}}{\alpha_{12}+\alpha_{10} x_{n}+\alpha_{11} x_{n-1}},
$$

where $\alpha_{s}(s=10,11,12)$ and $x_{s}(s=-1,0)$ are the positive real numbers. More precisely, .Bozkurt [2] has explored the local asymptotic stability of the equilibrium point by linearized stability theorem, gasymptotic stability behavior by Lyapunov function, and semicycle analysis of positive solutions of the exponential difference equation, which is depicted in (2). Finally, theoretical results are verified numerically. Motivated from the aforementioned studies, here our purpose is to explore the global dynamical properties of the following $(2,3)$-type exponential systems, that is the extension of the work of Bozkurt [2]:

$$
\begin{aligned}
& x_{n+1}=\frac{\alpha_{10} e^{-y_{n}}+\alpha_{11} e^{-y_{n-1}}}{\alpha_{12}+\alpha_{10} y_{n}+\alpha_{11} y_{n-1}}, \\
& y_{n+1}=\frac{\alpha_{13} e^{-z_{n}}+\alpha_{14} e^{-z_{n-1}}}{\alpha_{15}+\alpha_{13} z_{n}+\alpha_{14} z_{n-1}}, \\
& z_{n+1}=\frac{\alpha_{16} e^{-x_{n}}+\alpha_{17} e^{-x_{n-1}}}{\alpha_{18}+\alpha_{16} x_{n}+\alpha_{17} x_{n-1}},
\end{aligned}
$$




$$
\begin{aligned}
& x_{n+1}=\frac{\alpha_{19} e^{-z_{n}}+\alpha_{20} e^{-z_{n-1}}}{\alpha_{21}+\alpha_{19} z_{n}+\alpha_{20} z_{n-1}}, \\
& y_{n+1}=\frac{\alpha_{22} e^{-x_{n}}+\alpha_{23} e^{-x_{n-1}}}{\alpha_{24}+\alpha_{22} x_{n}+\alpha_{23} x_{n-1}} \text {, } \\
& z_{n+1}=\frac{\alpha_{25} e^{-y_{n}}+\alpha_{26} e^{-y_{n-1}}}{\alpha_{27}+\alpha_{25} y_{n}+\alpha_{26} y_{n-1}}, \\
& x_{n+1}=\frac{\alpha_{28} e^{-x_{n}}+\alpha_{29} e^{-x_{n-1}}}{\alpha_{30}+\alpha_{28} x_{n}+\alpha_{29} x_{n-1}}, \\
& y_{n+1}=\frac{\alpha_{31} e^{-y_{n}}+\alpha_{32} e^{-y_{n-1}}}{\alpha_{33}+\alpha_{31} z_{n}+\alpha_{32} z_{n-1}} \text {, } \\
& z_{n+1}=\frac{\alpha_{34} e^{-z_{n}}+\alpha_{35} e^{-z_{n-1}}}{\alpha_{36}+\alpha_{34} y_{n}+\alpha_{35} y_{n-1}}, \\
& x_{n+1}=\frac{\alpha_{37} e^{-x_{n}}+\alpha_{38} e^{-x_{n-1}}}{\alpha_{39}+\alpha_{37} y_{n}+\alpha_{38} y_{n-1}}, \\
& y_{n+1}=\frac{\alpha_{40} e^{-y_{n}}+\alpha_{41} e^{-y_{n-1}}}{\alpha_{42}+\alpha_{40} x_{n}+\alpha_{41} x_{n-1}}, \\
& z_{n+1}=\frac{\alpha_{43} e^{-z_{n}}+\alpha_{44} e^{-z_{n-1}}}{\alpha_{45}+\alpha_{43} z_{n}+\alpha_{44} z_{n-1}} \text {, } \\
& x_{n+1}=\frac{\alpha_{46} e^{-x_{n}}+\alpha_{47} e^{-x_{n-1}}}{\alpha_{48}+\alpha_{46} z_{n}+\alpha_{47} z_{n-1}}, \\
& y_{n+1}=\frac{\alpha_{49} e^{-y_{n}}+\alpha_{50} e^{-y_{n-1}}}{\alpha_{51}+\alpha_{49} y_{n}+\alpha_{50} y_{n-1}} \text {, } \\
& z_{n+1}=\frac{\alpha_{52} e^{-z_{n}}+\alpha_{53} e^{-z_{n-1}}}{\alpha_{54}+\alpha_{52} x_{n}+\alpha_{53} x_{n-1}}, \\
& x_{n+1}=\frac{\alpha_{55} e^{-z_{n}}+\alpha_{56} e^{-z_{n-1}}}{\alpha_{57}+\alpha_{55} x_{n}+\alpha_{56} x_{n-1}}, \\
& y_{n+1}=\frac{\alpha_{58} e^{-x_{n}}+\alpha_{59} e^{-x_{n-1}}}{\alpha_{60}+\alpha_{58} y_{n}+\alpha_{59} y_{n-1}} \text {, } \\
& z_{n+1}=\frac{\alpha_{61} e^{-y_{n}}+\alpha_{62} e^{-y_{n-1}}}{\alpha_{63}+\alpha_{61} z_{n}+\alpha_{62} z_{n-1}},
\end{aligned}
$$

where $\alpha_{s}(s=10, \ldots, 63)$ and $x_{s}, y_{s}, z_{s}(s=-1,0)$ are the positive real numbers.

The rest of the paper is organized as follows: in Section 2 , we explore that every positive solution of systems (3)-(8) is bounded and persists, whereas construction of an invariant rectangle is explored in Section 3. In Section 4 , we explore the existence as well as uniqueness of the positive equilibrium point of systems (3)-(8). In Section 5, we explore the local dynamical properties about the unique positive equilibrium point of systems (3)-(8). In Section 6, we explore global dynamics about the positive equilibrium by the discrete-time Lyapunov function. We study the rate of convergence in Section 7, whereas discussion along with numerical simulations is presented in Section 8.

\section{Boundedness and Persistence of Systems (3) $-(8)$}

Theorem 1. Every positive solution $\left\{\Omega_{n}\right\}_{n=-1}^{\infty}$ of systems (3)-(8) is bounded and persists.

Proof. (i) If $\left\{\Omega_{n}\right\}_{n=-1}^{\infty}$ is a positive solution of (3), then

$$
\begin{aligned}
x_{n} \leq \frac{\alpha_{10}+\alpha_{11}}{\alpha_{12}} & =U_{1}, \\
y_{n} \leq \frac{\alpha_{13}+\alpha_{14}}{\alpha_{15}} & =U_{2}, \\
z_{n} \leq \frac{\alpha_{16}+\alpha_{17}}{\alpha_{18}} & =U_{3}, \\
n & =1,2, \ldots
\end{aligned}
$$

From (3) and (9), one gets

$$
\begin{gathered}
x_{n} \geq \frac{\left(\alpha_{10}+\alpha_{11}\right) e^{-\left(\left(\alpha_{13}+\alpha_{14}\right) / \alpha_{15}\right)}}{\alpha_{10}+\left(\alpha_{11}+\alpha_{12}\right)\left(\left(\alpha_{13}+\alpha_{14}\right) / \alpha_{15}\right)}=L_{1}, \\
y_{n} \geq \frac{\left(\alpha_{13}+\alpha_{14}\right) e^{-\left(\left(\alpha_{16}+\alpha_{17}\right) / \alpha_{18}\right)}}{\alpha_{15}+\left(\alpha_{13}+\alpha_{14}\right)\left(\left(\alpha_{16}+\alpha_{17}\right) / \alpha_{18}\right)}=L_{2}, \\
z_{n} \geq \frac{\left(\alpha_{16}+\alpha_{17}\right) e^{-\left(\left(\alpha_{10}+\alpha_{11}\right) / \alpha_{12}\right)}}{\alpha_{18}+\left(\alpha_{16}+\alpha_{17}\right)\left(\left(\alpha_{10}+\alpha_{11}\right) / \alpha_{12}\right)}=L_{3}, \\
n=2,3, \ldots
\end{gathered}
$$

So, from (9) and (10), one has

$$
\begin{aligned}
& L_{1} \leq x_{n} \leq U_{1}, \\
& L_{2} \leq y_{n} \leq U_{2}, \\
& L_{3} \leq z_{n} \leq U_{3}, \\
& \quad n=3,4, \ldots .
\end{aligned}
$$

(ii) If $\left\{\Omega_{n}\right\}_{n=-1}^{\infty}$ is a positive solution of (4), then

$$
\begin{aligned}
x_{n} \leq \frac{\alpha_{19}+\alpha_{20}}{\alpha_{21}} & =U_{4}, \\
y_{n} \leq \frac{\alpha_{22}+\alpha_{23}}{\alpha_{24}} & =U_{5}, \\
z_{n} \leq \frac{\alpha_{25}+\alpha_{26}}{\alpha_{27}} & =U_{6}, \\
n & =1,2, \ldots
\end{aligned}
$$

From (4) and (12), one gets 


$$
\begin{gathered}
x_{n} \geq \frac{\left(\alpha_{19}+\alpha_{20}\right) e^{-\left(\left(\alpha_{25}+\alpha_{26}\right) / \alpha_{27}\right)}}{\alpha_{21}+\left(\alpha_{19}+\alpha_{20}\right)\left(\left(\alpha_{25}+\alpha_{26}\right) / \alpha_{27}\right)}=L_{4}, \\
y_{n} \geq \frac{\left(\alpha_{22}+\alpha_{23}\right) e^{-\left(\left(\alpha_{19}+\alpha_{20}\right) / \alpha_{21}\right)}}{\alpha_{24}+\left(\alpha_{22}+\alpha_{23}\right)\left(\left(\alpha_{19}+\alpha_{20}\right) / \alpha_{21}\right)}=L_{5}, \\
z_{n} \geq \frac{\left(\alpha_{25}+\alpha_{26}\right) e^{-\left(\left(\alpha_{22}+\alpha_{23}\right) / \alpha_{24}\right)}}{\alpha_{27}+\left(\alpha_{25}+\alpha_{26}\right)\left(\left(\alpha_{22}+\alpha_{23}\right) / \alpha_{24}\right)}=L_{6}, \\
n=2,3, \ldots
\end{gathered}
$$

So, from (12) and (13), one gets

$$
\begin{aligned}
& L_{4} \leq x_{n} \leq U_{4}, \\
& L_{5} \leq y_{n} \leq U_{5}, \\
& L_{6} \leq z_{n} \leq U_{6}, \\
& \quad n=3,4, \ldots .
\end{aligned}
$$

(iii) If $\left\{\Omega_{n}\right\}_{n=-1}^{\infty}$ is a positive solution of (5), then

$$
\begin{aligned}
x_{n} \leq \frac{\alpha_{28}+\alpha_{29}}{\alpha_{30}} & =U_{7}, \\
y_{n} \leq \frac{\alpha_{31}+\alpha_{32}}{\alpha_{33}} & =U_{8}, \\
z_{n} \leq \frac{\alpha_{34}+\alpha_{35}}{\alpha_{36}} & =U_{9}, \\
n & =1,2, \ldots
\end{aligned}
$$

From (5) and (15), one gets

$$
\begin{gathered}
x_{n} \geq \frac{\left(\alpha_{28}+\alpha_{29}\right) e^{-\left(\left(\alpha_{28}+\alpha_{29}\right) / \alpha_{30}\right)}}{\alpha_{30}+\left(\alpha_{28}+\alpha_{29}\right)\left(\left(\alpha_{28}+\alpha_{29}\right) / \alpha_{30}\right)}=L_{7}, \\
y_{n} \geq \frac{\left(\alpha_{31}+\alpha_{32}\right) e^{-\left(\left(\alpha_{31}+\alpha_{32}\right) / \alpha_{33}\right)}}{\alpha_{33}+\left(\alpha_{31}+\alpha_{32}\right)\left(\left(\alpha_{34}+\alpha_{35}\right) / \alpha_{36}\right)}=L_{8}, \\
z_{n} \geq \frac{\left(\alpha_{34}+\alpha_{35}\right) e^{-\left(\left(\alpha_{34}+\alpha_{35}\right) / \alpha_{35}\right)}}{\alpha_{36}+\left(\alpha_{34}+\alpha_{35}\right)\left(\left(\alpha_{31}+\alpha_{32}\right) / \alpha_{33}\right)}=L_{9}, \\
n=2,3, \ldots
\end{gathered}
$$

So, from (15) and (16), one gets

$$
\begin{aligned}
L_{7} \leq x_{n} & \leq U_{7}, \\
L_{8} \leq y_{n} & \leq U_{8}, \\
L_{9} \leq z_{n} & \leq U_{9}, \\
& n=3,4, \ldots .
\end{aligned}
$$

(iv) If $\left\{\Omega_{n}\right\}_{n=-1}^{\infty}$ is a positive solution of (6), then

$$
\begin{aligned}
x_{n} \leq \frac{\alpha_{37}+\alpha_{38}}{\alpha_{39}} & =U_{10}, \\
y_{n} \leq \frac{\alpha_{40}+\alpha_{41}}{\alpha_{42}} & =U_{11}, \\
z_{n} \leq \frac{\alpha_{43}+\alpha_{44}}{\alpha_{45}} & =U_{12}, \\
n & =1,2, \ldots
\end{aligned}
$$

From (6) and (18), one gets

$$
\begin{gathered}
x_{n} \geq \frac{\left(\alpha_{37}+\alpha_{38}\right) e^{-\left(\left(\alpha_{37}+\alpha_{38}\right) / \alpha_{39}\right)}}{\alpha_{39}+\left(\alpha_{37}+\alpha_{38}\right)\left(\left(\alpha_{40}+\alpha_{41}\right) / \alpha_{42}\right)}=L_{10}, \\
y_{n} \geq \frac{\left(\alpha_{40}+\alpha_{41}\right) e^{-\left(\left(\alpha_{40}+\alpha_{41}\right) / \alpha_{42}\right)}}{\alpha_{42}+\left(\alpha_{40}+\alpha_{41}\right)\left(\left(\alpha_{37}+\alpha_{38}\right) / \alpha_{39}\right)}=L_{11}, \\
z_{n} \geq \frac{\left(\alpha_{43}+\alpha_{44}\right) e^{-\left(\left(\alpha_{43}+\alpha_{44}\right) / \alpha_{45}\right)}}{\alpha_{45}+\left(\alpha_{43}+\alpha_{44}\right)\left(\left(\alpha_{43}+\alpha_{44}\right) / \alpha_{45}\right)}=L_{12}, \\
n=2,3, \ldots
\end{gathered}
$$

So, from (18) and (19), one gets

$$
\begin{aligned}
L_{10} \leq x_{n} & \leq U_{10}, \\
L_{11} \leq y_{n} & \leq U_{11}, \\
L_{12} \leq z_{n} & \leq U_{12}, \\
n & =3,4, \ldots
\end{aligned}
$$

(v) If $\left\{\Omega_{n}\right\}_{n=-1}^{\infty}$ is a positive solution of (7), then

$$
\begin{gathered}
x_{n} \leq \frac{\alpha_{46}+\alpha_{47}}{\alpha_{48}}=U_{13}, \\
y_{n} \leq \frac{\alpha_{49}+\alpha_{50}}{\alpha_{51}}=U_{14}, \\
z_{n} \leq \frac{\alpha_{52}+\alpha_{53}}{\alpha_{54}}=U_{15}, \\
n=1,2, \ldots
\end{gathered}
$$

From (7) and (21), one gets

$$
\begin{aligned}
& x_{n} \geq \frac{\left(\alpha_{46}+\alpha_{47}\right) e^{-\left(\left(\alpha_{46}+\alpha_{47}\right) / \alpha_{48}\right)}}{\alpha_{48}+\left(\alpha_{46}+\alpha_{47}\right)\left(\left(\alpha_{52}+\alpha_{53}\right) / \alpha_{54}\right)}=L_{13}, \\
& y_{n} \geq \frac{\left(\alpha_{49}+\alpha_{50}\right) e^{-\left(\left(\alpha_{49}+\alpha_{50}\right) / \alpha_{51}\right)}}{\alpha_{51}+\left(\alpha_{49}+\alpha_{50}\right)\left(\left(\alpha_{49}+\alpha_{50}\right) / \alpha_{51}\right)}=L_{14}, \\
& z_{n} \geq \frac{\left(\alpha_{52}+\alpha_{53}\right) e^{-\left(\left(\alpha_{52}+\alpha_{53}\right) / \alpha_{54}\right)}}{\alpha_{54}+\left(\alpha_{52}+\alpha_{53}\right)\left(\left(\alpha_{46}+\alpha_{47}\right) / \alpha_{48}\right)}=L_{15},
\end{aligned}
$$

$$
n=2,3, \ldots
$$


So, from (21) and (22), one gets

$$
\begin{aligned}
L_{13} \leq x_{n} & \leq U_{13}, \\
L_{14} \leq y_{n} & \leq U_{14}, \\
L_{15} \leq z_{n} & \leq U_{15}, \\
n & =3,4, \ldots .
\end{aligned}
$$

(vi) If $\left\{\Omega_{n}\right\}_{n=-1}^{\infty}$ is a positive solution of (8), then

$$
\begin{gathered}
x_{n} \leq \frac{\alpha_{55}+\alpha_{56}}{\alpha_{57}}=U_{16}, \\
y_{n} \leq \frac{\alpha_{58}+\alpha_{59}}{\alpha_{60}}=U_{17}, \\
z_{n} \leq \frac{\alpha_{61}+\alpha_{62}}{\alpha_{63}}=U_{18}, \\
n=1,2, \ldots
\end{gathered}
$$

From (8) and (24), one gets

$$
\begin{gathered}
x_{n} \geq \frac{\left(\alpha_{55}+\alpha_{56}\right) e^{-\left(\left(\alpha_{61}+\alpha_{62}\right) / \alpha_{63}\right)}}{\alpha_{57}+\left(\alpha_{55}+\alpha_{56}\right)\left(\left(\alpha_{55}+\alpha_{56}\right) / \alpha_{57}\right)}=L_{16}, \\
y_{n} \geq \frac{\left(\alpha_{58}+\alpha_{59}\right) e^{-\left(\left(\alpha_{55}+\alpha_{56}\right) / \alpha_{57}\right)}}{\alpha_{60}+\left(\alpha_{58}+\alpha_{59}\right)\left(\left(\alpha_{58}+\alpha_{59}\right) / \alpha_{60}\right)}=L_{17}, \\
z_{n} \geq \frac{\left(\alpha_{61}+\alpha_{62}\right) e^{-\left(\left(\alpha_{58}+\alpha_{59}\right) / \alpha_{60}\right)}}{\alpha_{63}+\left(\alpha_{61}+\alpha_{62}\right)\left(\left(\alpha_{61}+\alpha_{62}\right) / \alpha_{63}\right)}=L_{18}, \\
n=2,3, \ldots
\end{gathered}
$$

So, from (24) and (25), one gets

$$
\begin{aligned}
L_{16} \leq x_{n} & \leq U_{16}, \\
L_{17} \leq y_{n} & \leq U_{17}, \\
L_{18} \leq z_{n} & \leq U_{18}, \\
n & =3,4, \ldots .
\end{aligned}
$$

\section{Existence of Invariant Rectangle of Systems (3) $-(8)$}

Theorem 2. If $\left\{\Omega_{n}\right\}_{n=-1}^{\infty}$ is a positive solution of systems (3)-(8), then their corresponding invariant rectangles, respectively, are $\left[L_{1}, U_{1}\right] \times\left[L_{2}, U_{2}\right] \times\left[L_{3}, U_{3}\right],\left[L_{4}, U_{4}\right] \times$ $\left[L_{5}, U_{5}\right] \times\left[L_{6}, U_{6}\right],\left[L_{7}, U_{7}\right] \times\left[L_{8}, U_{8}\right] \times\left[L_{9}, U_{9}\right],\left[L_{10}, U_{10}\right]$ $\times\left[L_{11}, U_{11}\right] \times\left[L_{12}, U_{12}\right],\left[L_{13}, U_{13}\right] \times\left[L_{14}, U_{14}\right] \times\left[L_{15}, U_{15}\right]$, and $\left[L_{16}, U_{16}\right] \times\left[L_{17}, U_{17}\right] \times\left[L_{18}, U_{18}\right]$.

Proof. If $\left\{\Omega_{n}\right\}_{n=-1}^{\infty}$ is a positive solution with $x_{0}, x_{-1} \in\left[L_{1}, U_{1}\right], y_{0}, y_{-1} \in\left[L_{2}, U_{2}\right]$, and $z_{0}, z_{-1} \in\left[L_{3}, U_{3}\right]$, then from (3), one has

$$
\begin{aligned}
& x_{1}=\frac{\alpha_{10} e^{-y_{0}}+\alpha_{11} e^{-y_{-1}}}{\alpha_{12}+\alpha_{10} y_{0}+\alpha_{11} y_{-1}} \leq \frac{\alpha_{10}+\alpha_{11}}{\alpha_{12}}, \\
& x_{1}=\frac{\alpha_{10} e^{-y_{0}}+\alpha_{11} e^{-y_{-1}}}{\alpha_{12}+\alpha_{10} y_{0}+\alpha_{11} y_{-1}} \geq \frac{\left(\alpha_{10}+\alpha_{11}\right) e^{-\left(\left(\alpha_{13}+\alpha_{14}\right) / \alpha_{15}\right)}}{\alpha_{12}+\left(\alpha_{10}+\alpha_{11}\right)\left(\left(\alpha_{13}+\alpha_{14}\right) / \alpha_{15}\right)}, \\
& y_{1}=\frac{\alpha_{13} e^{-z_{0}}+\alpha_{14} e^{-z_{-1}}}{\alpha_{15}+\alpha_{13} z_{0}+\alpha_{14} z_{-1}} \leq \frac{\alpha_{13}+\alpha_{14}}{\alpha_{15}}, \\
& y_{1}=\frac{\alpha_{13} e^{-z_{0}}+\alpha_{14} e^{-z_{-1}}}{\alpha_{15}+\alpha_{13} z_{0}+\alpha_{14} z_{-1}} \geq \frac{\left(\alpha_{13}+\alpha_{14}\right) e^{-\left(\left(\alpha_{16}+\alpha_{17}\right) / \alpha_{18}\right)}}{\alpha_{15}+\left(\alpha_{13}+\alpha_{14}\right)\left(\left(\alpha_{16}+\alpha_{17}\right) / \alpha_{18}\right)}, \\
& z_{1}=\frac{\alpha_{16} e^{-x_{0}}+\alpha_{17} e^{-x_{-1}}}{\alpha_{18}+\alpha_{16} x_{0}+\alpha_{17} x_{-1}} \leq \frac{\alpha_{16}+\alpha_{17}}{\alpha_{18}}, \\
& z_{1}=\frac{\alpha_{16} e^{-x_{0}}+\alpha_{17} e^{-x_{-1}}}{\alpha_{18}+\alpha_{16} x_{0}+\alpha_{17} x_{-1}} \geq \frac{\left(\alpha_{16}+\alpha_{17}\right) e^{-\left(\left(\alpha_{10}+\alpha_{11}\right) / \alpha_{12}\right)}}{\alpha_{18}+\left(\alpha_{16}+\alpha_{17}\right)\left(\left(\alpha_{10}+\alpha_{11}\right) / \alpha_{12}\right)} .
\end{aligned}
$$

Hence (27) then implies that $x_{1} \in\left[L_{1}, U_{1}\right]$, $y_{1} \in\left[L_{2}, U_{2}\right]$, and $z_{1} \in\left[L_{3}, U_{3}\right]$. Finally from (3), it is easy to establish that $x_{k+1} \in\left[L_{1}, U_{1}\right]$ (resp., $y_{k+1} \in\left[L_{2}, U_{2}\right]$ and $\left.z_{k+1} \in\left[L_{3}, U_{3}\right]\right)$ if $x_{k} \in\left[L_{1}, U_{1}\right]$ (resp., $y_{k} \in\left[L_{2}, U_{2}\right]$ and $\left.z_{k} \in\left[L_{3}, U_{3}\right]\right)$.

Remark 1. In a similar way, one can prove the invariant rectangle for systems (4)-(8).

\section{Existence as well as Uniqueness of Positive Fixed Point of Systems (3) $-(8)$}

Existence as well as uniqueness of a positive fixed point of systems (3)-(8) is explored in this section, as follows.

\section{Theorem 3.}

(i) System (3) has a unique positive fixed point: $\Upsilon_{1}=$ $(\bar{x}, \bar{y}, \bar{z}) \in\left[L_{1}, U_{1}\right] \times\left[L_{2}, U_{2}\right] \times\left[L_{3}, U_{3}\right]$ if

$$
\begin{aligned}
& \frac{\left(\alpha_{13}+\alpha_{14}\right) e^{-L_{1}}\left(\left(U_{1}+1\right)\left(\alpha_{13}+\alpha_{14}\right)+\alpha_{15}\right)\left(\alpha_{10}+\alpha_{11}\right) e^{-\left(\left(\left(\alpha_{13}+\alpha_{14}\right) e^{-U_{1}}\right) /\left(\alpha_{15}+\left(\alpha_{13}+\alpha_{14}\right) L_{1}\right)\right)}}{\left(\alpha_{15}+\left(\alpha_{13}+\alpha_{14}\right) z\right)^{2}\left(\alpha_{18}+\left(\alpha_{16}+\alpha_{17}\right)\right)^{2}} \\
& \times \frac{\left(\left(\alpha_{10}+\alpha_{11}\right)\left(\left(\left(\left(\alpha_{13}+\alpha_{14}\right) e^{-L_{1}}\right) /\left(\alpha_{15}+\left(\alpha_{13}+\alpha_{14}\right) L_{1}\right)\right)+1\right)+\alpha_{12}\right)}{\left(\alpha_{12}+\left(\alpha_{10}+\alpha_{11}\right)\left(\left(\left(\alpha_{13}+\alpha_{14}\right) e^{-L_{1}}\right) /\left(\alpha_{15}+\left(\alpha_{13}+\alpha_{14}\right) L_{1}\right)\right)\right)^{2}}\left(\alpha_{16}+\alpha_{17}\right)
\end{aligned}
$$

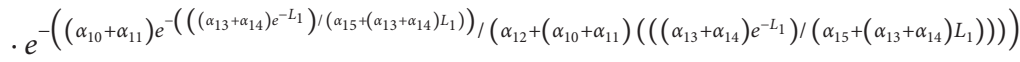

$$
\begin{aligned}
& \times\left(\left(\alpha_{16}+\alpha_{17}\right)\left(\Lambda_{1}+1\right)+\alpha_{18}\right)<\Lambda_{1}^{2},
\end{aligned}
$$


where

$$
\Lambda_{1}=\frac{\left(\alpha_{10}+\alpha_{11}\right) e^{-\left(\left(\left(\alpha_{13}+\alpha_{14}\right) e^{-U_{3}}\right) /\left(\alpha_{15}+\left(\alpha_{13}+\alpha_{14}\right) L_{3}\right)\right)}}{\alpha_{12}+\left(\alpha_{10}+\alpha_{11}\right)\left(\left(\left(\alpha_{13}+\alpha_{14}\right) e^{-L_{3}}\right) /\left(\alpha_{15}+\left(\alpha_{13}+\alpha_{14}\right) L_{3}\right)\right)} ;
$$

(ii) System (4) has a unique positive fixed point: $\Upsilon_{2}=$ $(\bar{x}, \bar{y}, \bar{z}) \in\left[L_{4}, U_{4}\right] \times\left[L_{5}, U_{5}\right] \times\left[L_{6}, U_{6}\right]$ if

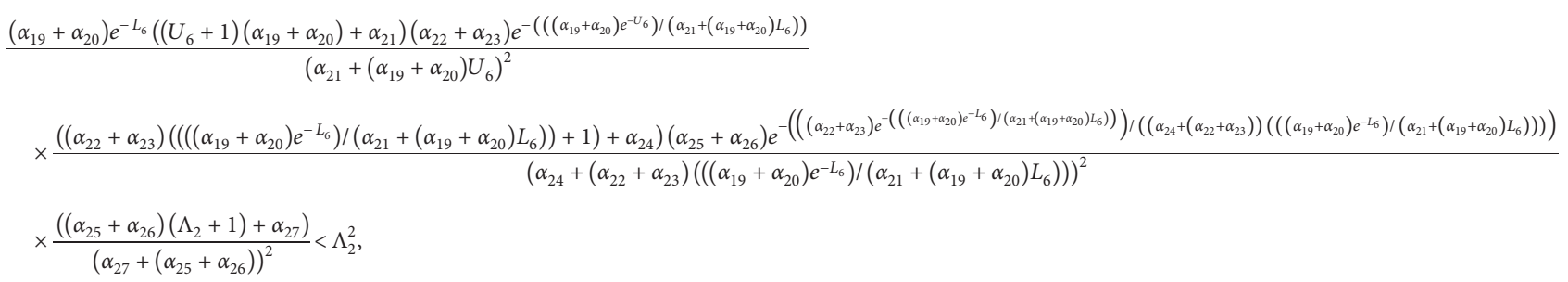

where

$$
\Lambda_{2}=\frac{\left(\alpha_{22}+\alpha_{23}\right) e^{-\left(\left(\left(\alpha_{19}+\alpha_{20}\right) e^{-U_{6}}\right) /\left(\alpha_{21}+\left(\alpha_{19}+\alpha_{20}\right) L_{6}\right)\right)}}{\left.\alpha_{24}+\left(\alpha_{22}+\alpha_{23}\right)\left(\left(\alpha_{19}+\alpha_{20}\right) e^{-L_{6}}\right) /\left(\alpha_{21}+\left(\alpha_{19}+\alpha_{20}\right) L_{6}\right)\right)} ;
$$

(iii) System (5) has a unique positive fixed point: $\Upsilon_{3}=$ $(\bar{x}, \bar{y}, \bar{z}) \in\left[L_{7}, U_{7}\right] \times\left[L_{8}, U_{8}\right] \times\left[L_{9}, U_{9}\right]$ if

$$
\begin{gathered}
\frac{e^{-\left(\left(e^{-U_{8} / L_{8}}\right)-\left(\alpha_{33} /\left(\alpha_{31}+\alpha_{32}\right)\right)\right)} e^{-L_{8}}\left(U_{8}+1\right)\left(\left(e^{-L_{8}} / L_{8}\right)-\left(\alpha_{33} /\left(\alpha_{31}+\alpha_{32}\right)\right)+1\right)}{\left(\left(e^{-L_{8} / L_{8}}\right)-\left(\alpha_{33} /\left(\alpha_{31}+\alpha_{32}\right)\right)\right)^{2} U_{8}^{2}} \\
\cdot e^{-\left(\left(e^{-\left(\left(e^{-L_{8} / U_{8}}\right)-\left(\alpha_{33} /\left(\alpha_{31}+\alpha_{32}\right)\right)\right)} /\left(e^{\left.-L_{8} / L_{8}\right)}-\left(\alpha_{33} /\left(\alpha_{31}+\alpha_{32}\right)\right)\right)-\left(\alpha_{36} /\left(\alpha_{34}+\alpha_{35}\right)\right)\right)\right.}<\Lambda_{3}^{2},
\end{gathered}
$$

where

$\Lambda_{3}=\frac{e^{-\left(\left(e^{-U_{8} / L_{8}}\right)-\left(\alpha_{33} /\left(\alpha_{31}+\alpha_{32}\right)\right)\right)}}{\left(\left(e^{\left.-L_{8} / L_{8}\right)}-\left(\alpha_{33} /\left(\alpha_{31}+\alpha_{32}\right)\right)\right)\right.}-\frac{\alpha_{36}}{\alpha_{34}+\alpha_{35}} ;$ (iv) System (6) has a unique positive fixed point: $\Upsilon_{4}=$ $(\bar{x}, \bar{y}, \bar{z}) \in\left[L_{10}, U_{10}\right] \times\left[L_{11}, U_{11}\right] \times\left[L_{12}, U_{12}\right]$ if

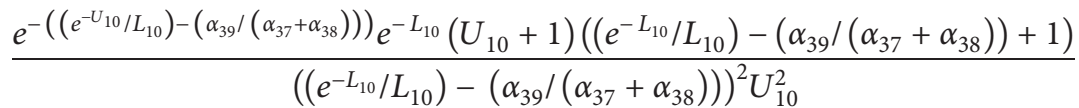

$$
\begin{aligned}
& \cdot e^{-\left(\left(e^{-\left(\left(e^{-L} 10 / U_{10}\right)-\left(\alpha_{39} /\left(\alpha_{37}+\alpha_{38}\right)\right)\right)} /\left(e^{-L_{10} / L_{10}}\right)-\left(\alpha_{39} /\left(\alpha_{37}+\alpha_{38}\right)\right)\right)-\left(\alpha_{42} /\left(\alpha_{40}+\alpha_{41}\right)\right)\right)}<\Lambda_{4}^{2} \text {, }
\end{aligned}
$$


where

$\Lambda_{4}=\frac{e^{-\left(\left(e^{-U_{10} / L_{10}}\right)-\left(\alpha_{39} /\left(\alpha_{37}+\alpha_{38}\right)\right)\right)}}{\left(\left(e^{\left.-L_{10} / L_{10}\right)}-\left(\alpha_{39} /\left(\alpha_{37}+\alpha_{38}\right)\right)\right)\right.}-\frac{\alpha_{42}}{\alpha_{40}+\alpha_{41}}$ (v) System (7) has a unique positive fixed point: $\Upsilon_{5}=$ $(\bar{x}, \bar{y}, \bar{z}) \in\left[L_{13}, U_{13}\right] \times\left[L_{14}, U_{14}\right] \times\left[L_{15}, U_{15}\right]$ if

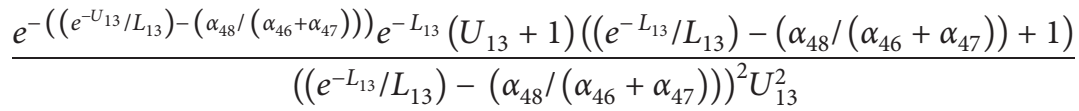

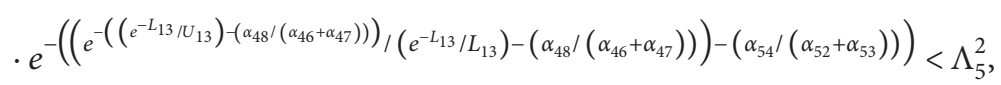

where

$$
\Lambda_{5}=\frac{e^{-\left(\left(e^{-U_{13} / L_{13}}\right)-\left(\alpha_{48} /\left(\alpha_{46}+\alpha_{47}\right)\right)\right)}}{\left(\left(\left(e^{\left.-L_{13} / L_{13}\right)}-\left(\alpha_{48} /\left(\alpha_{46}+\alpha_{47}\right)\right)\right)\right)\right.}-\frac{\alpha_{54}}{\alpha_{52}+\alpha_{53}}
$$

(vi) System (8) has a unique positive fixed point: $\Upsilon_{6}=$ $(\bar{x}, \bar{y}, \bar{z}) \in\left[L_{16}, U_{16}\right] \times\left[L_{17}, U_{17}\right] \times\left[L_{18}, U_{18}\right]$ if

$$
\begin{aligned}
& \frac{\left(\alpha_{57}+2 U_{16}\left(\alpha_{55}+\alpha_{56}\right)\right)\left(\alpha_{63}+2 U_{16}\left(\alpha_{61}+\alpha_{62}\right) \ln \left(\left(\alpha_{55}+\alpha_{56}\right) /\left(L_{16}\left(\alpha_{57}+\left(\alpha_{55}+\alpha_{56}\right) L_{16}\right)\right)\right)\right)}{\left(\alpha_{63} \ln \left(\alpha_{55}+\alpha_{56}\right) / L_{16}\left(\alpha_{57}+\left(\alpha_{55}+\alpha_{56}\right) L_{16}\right)+\left(\alpha_{61}+\alpha_{62}\right)\left(\ln \left(\left(\alpha_{55}+\alpha_{56}\right) /\left(L_{16}\left(\alpha_{57}+\left(\alpha_{55}+\alpha_{56}\right) L_{16}\right)\right)\right)\right)^{2}\right)} \\
& \times\left(\frac{\alpha_{57}+2 U_{16}\left(\alpha_{55}+\alpha_{56}\right)\left(\alpha_{63}+2\left(\alpha_{61}+\alpha_{62}\right)\right) \ln \left(\left(\alpha_{55}+\alpha_{56}\right) /\left(L_{16}\left(\alpha_{57}+\left(\alpha_{55}+\alpha_{56}\right) L_{16}\right)\right)\right)}{\alpha_{63} \ln \left(\left(\alpha_{55}+\alpha_{56}\right) /\left(L_{16}\left(\alpha_{57}+\left(\alpha_{55}+\alpha_{56}\right) L_{16}\right)\right)\right)}+\Lambda_{6}\right) \\
& \times \frac{\alpha_{60}\left(\alpha_{58}+\alpha_{59}\right)}{L_{16}\left(\alpha_{57}+\left(\alpha_{55}+\alpha_{56}\right) L_{16}\right)\left(\alpha_{60}+\left(\alpha_{58}+\alpha_{59}\right)\right)}<\Lambda_{6},
\end{aligned}
$$

where

$$
\Lambda_{6}=\ln \left(\frac{\alpha_{61}+\alpha_{62}}{\ln \left(\left(\alpha_{55}+\alpha_{56}\right) /\left(L_{16}\left(\alpha_{57}+\left(\alpha_{55}+\alpha_{56}\right) L_{16}\right)\right)\right)\left(\alpha_{63}+\left(\alpha_{61}+\alpha_{62}\right) \ln \left(\left(\alpha_{55}+\alpha_{56}\right) /\left(L_{16}\left(\alpha_{57}+\left(\alpha_{55}+\alpha_{56}\right) L_{16}\right)\right)\right)\right.}\right) .
$$

Proof. (i) From (3), one has

$$
\begin{aligned}
& x=\frac{\left(\alpha_{10}+\alpha_{11}\right) e^{-y}}{\alpha_{12}+\left(\alpha_{10}+\alpha_{11}\right) y}, \\
& y=\frac{\left(\alpha_{13}+\alpha_{14}\right) e^{-z}}{\alpha_{15}+\left(\alpha_{13}+\alpha_{14}\right) z}, \\
& z=\frac{\left(\alpha_{16}+\alpha_{17}\right) e^{-x}}{\alpha_{18}+\left(\alpha_{16}+\alpha_{17}\right) x} .
\end{aligned}
$$

From (40), one gets

$$
\begin{aligned}
& z=\frac{\left(\alpha_{16}+\alpha_{17}\right) e^{-x}}{\alpha_{18}+\left(\alpha_{16}+\alpha_{17}\right) x}, \\
& x=\frac{\left(\alpha_{10}+\alpha_{11}\right) e^{-y}}{\alpha_{12}+\left(\alpha_{10}+\alpha_{11}\right) y}, \\
& y=\frac{\left(\alpha_{13}+\alpha_{14}\right) e^{-z}}{\alpha_{15}+\left(\alpha_{13}+\alpha_{14}\right) z} .
\end{aligned}
$$

From (41), setting

$$
\begin{aligned}
& y=g(z)=\frac{\left(\alpha_{13}+\alpha_{14}\right) e^{-z}}{\alpha_{15}+\left(\alpha_{13}+\alpha_{14}\right) z}, \\
& x=f(y)=\frac{\left(\alpha_{10}+\alpha_{11}\right) e^{-y}}{\alpha_{12}+\left(\alpha_{10}+\alpha_{11}\right) y} .
\end{aligned}
$$


Denote

$$
F(z):=\frac{\left(\alpha_{16}+\alpha_{17}\right) e^{-k(z)}}{\alpha_{18}+\left(\alpha_{16}+\alpha_{17}\right) k(z)}-z
$$

where

$$
\begin{aligned}
k(z) & :=x=f(g(z)), \\
& =f\left(\frac{\left(\alpha_{13}+\alpha_{14}\right) e^{-z}}{\alpha_{15}+\left(\alpha_{13}+\alpha_{14}\right) z}\right), \\
& =\frac{\left(\alpha_{10}+\alpha_{11}\right) e^{-\left(\left(\left(\alpha_{13}+\alpha_{14}\right) e^{-z}\right) /\left(\alpha_{15}+\left(\alpha_{13}+\alpha_{14}\right) z\right)\right)}}{\alpha_{12}+\left(\alpha_{10}+\alpha_{11}\right)\left(\left(\left(\alpha_{13}+\alpha_{14}\right) e^{-z}\right) /\left(\alpha_{15}+\left(\alpha_{13}+\alpha_{14}\right) z\right)\right)},
\end{aligned}
$$

and $z \in\left[L_{3}, U_{3}\right]$. Here, our finding is that $F(z)=0$ has a unique solution, where $z \in\left[L_{3}, U_{3}\right]$. From (43) and (44), one gets

$$
F^{\prime}(z)=-\frac{k^{\prime}(z)\left(\alpha_{16}+\alpha_{17}\right) e^{-k(z)}\left((k(z)+1)\left(\alpha_{16}+\alpha_{17}\right)+\alpha_{18}\right)}{\left(\alpha_{18}+\left(\alpha_{16}+\alpha_{17}\right) k(z)\right)^{2}}-1,
$$

where

$$
\begin{aligned}
k^{\prime}(z)= & -\frac{\left(\alpha_{13}+\alpha_{14}\right) e^{-z}\left((z+1)\left(\alpha_{13}+\alpha_{14}\right)+\alpha_{15}\right)\left(\alpha_{10}+\alpha_{11}\right) e^{-\left(\left(\left(\alpha_{13}+\alpha_{14}\right) e^{-z}\right) /\left(\alpha_{15}+\left(\alpha_{13}+\alpha_{14}\right) z\right)\right)}}{\left(\alpha_{15}+\left(\alpha_{13}+\alpha_{14}\right) z\right)^{2}\left(\alpha_{12}+\left(\alpha_{10}+\alpha_{11}\right)\left(\left(\left(\alpha_{13}+\alpha_{14}\right) e^{-z}\right) /\left(\alpha_{15}+\left(\alpha_{13}+\alpha_{14}\right) z\right)\right)\right)^{2}} \\
& \times\left(\left(\alpha_{10}+\alpha_{11}\right)\left(\frac{\left(\alpha_{13}+\alpha_{14}\right) e^{-z}}{\alpha_{15}+\left(\alpha_{13}+\alpha_{14}\right) z}+1\right)+\alpha_{12}\right) .
\end{aligned}
$$

Using (44) and (46) in (45), we obtain

$$
\begin{aligned}
& F^{\prime}(z)=\frac{\left(\alpha_{13}+\alpha_{14}\right) e^{-z}\left((z+1)\left(\alpha_{13}+\alpha_{14}\right)+\alpha_{15}\right)\left(\alpha_{10}+\alpha_{11}\right) e^{-\left(\left(\left(\alpha_{13}+\alpha_{14}\right) e^{-z}\right) /\left(\alpha_{15}+\left(\alpha_{13}+\alpha_{14}\right) z\right)\right)}}{\left(\alpha_{15}+\left(\alpha_{13}+\alpha_{14}\right) z\right)^{2}\left(\alpha_{18}+\left(\alpha_{16}+\alpha_{17}\right)\left(\left(\left(\alpha_{10}+\alpha_{11}\right) e^{-\left(\left(\left(\alpha_{13}+\alpha_{14}\right) e^{-z}\right) /\left(\alpha_{15}+\left(\alpha_{13}+\alpha_{14}\right) z\right)\right)}\right) /\left(\alpha_{12}+\left(\alpha_{10}+\alpha_{11}\right)\left(\left(\left(\alpha_{13}+\alpha_{14}\right) e^{-z}\right) /\left(\alpha_{15}+\left(\alpha_{13}+\alpha_{14}\right) z\right)\right)\right)\right)\right)^{2}} \times
\end{aligned}
$$

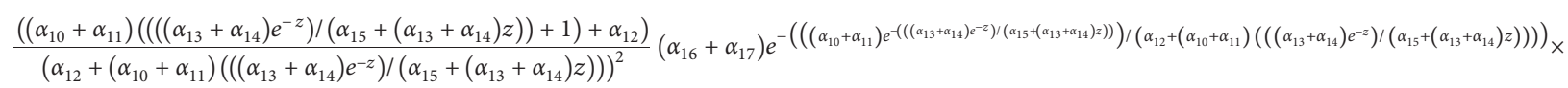

$$
\begin{aligned}
& \cdot\left(\left(\alpha_{16}+\alpha_{17}\right)\left(\frac{\left(\alpha_{10}+\alpha_{11}\right) e^{-\left(\left(\left(\alpha_{13}+\alpha_{14}\right) e^{-z}\right) /\left(\alpha_{15}+\left(\alpha_{13}+\alpha_{14}\right) z\right)\right)}}{\alpha_{12}+\left(\alpha_{10}+\alpha_{11}\right)\left(\left(\left(\alpha_{13}+\alpha_{14}\right) e^{-z}\right) /\left(\alpha_{15}+\left(\alpha_{13}+\alpha_{14}\right) z\right)\right)}+1\right)+\alpha_{18}\right)-1 \\
& \leq \frac{\left(\alpha_{13}+\alpha_{14}\right) e^{-L_{1}}\left(\left(U_{1}+1\right)\left(\alpha_{13}+\alpha_{14}\right)+\alpha_{15}\right)\left(\alpha_{10}+\alpha_{11}\right) e^{-\left(\left(\left(\alpha_{13}+\alpha_{14}\right) e^{-U_{1}}\right) /\left(\alpha_{15}+\left(\alpha_{13}+\alpha_{14}\right) L_{1}\right)\right)}}{\left(\alpha_{15}+\left(\alpha_{13}+\alpha_{14}\right) L_{1}\right)^{2}\left(\alpha_{18}+\left(\alpha_{16}+\alpha_{17}\right) \Lambda_{1}\right)^{2}} \times
\end{aligned}
$$

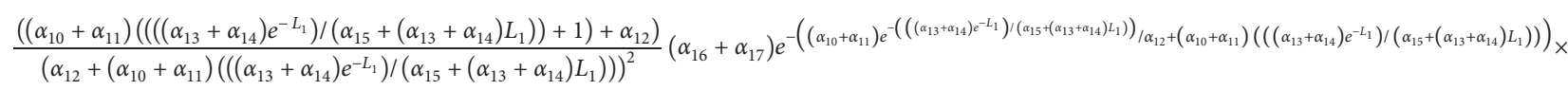

$$
\begin{aligned}
& \cdot\left(\left(\alpha_{16}+\alpha_{17}\right)\left(\Lambda_{1}+1\right)+\alpha_{18}\right)-1,
\end{aligned}
$$

where $\Lambda_{1}$ is depicted in (29). Now, assuming that if (28) along with (29) holds then from (47), one gets $F^{\prime}(z)<0$.

Remark 2. The proof of (ii)-(vi) is same as the proof of (i). So, it is omitted.

\section{Local Dynamics about Unique Positive Fixed Point of Systems (3)-(8)}

The local dynamics about $\Upsilon_{i}(i=1, \ldots, 6)$, respectively, of systems (3) to (8) is explored in this section, as follows. 
Theorem 4. For $\Upsilon_{1}$ of (3), the following holds:

(i) $\Upsilon_{1}$ is a sink if

$$
\begin{aligned}
& \frac{\alpha_{10} \alpha_{13}\left(\alpha_{14}+\alpha_{17}\right)\left(e^{-L_{1}}+L_{3}\right)\left(e^{-L_{2}}+L_{1}\right)\left(e^{-L_{3}}+L_{2}\right)}{\left(\alpha_{12}+\left(\alpha_{10}+\alpha_{11}\right) L_{2}\right)\left(\alpha_{15}+\left(\alpha_{13}+\alpha_{14}\right) L_{3}\right)\left(\alpha_{18}+\left(\alpha_{16}+\alpha_{17}\right) L_{1}\right)} \\
& +\frac{\alpha_{10} \alpha_{14}\left(\alpha_{13}+\alpha_{17}\right)\left(e^{-L_{1}}+L_{3}\right)\left(e^{-L_{2}}+L_{1}\right)\left(e^{-L_{3}}+L_{2}\right)}{\left(\alpha_{12}+\left(\alpha_{10}+\alpha_{11}\right) L_{2}\right)\left(\alpha_{15}+\left(\alpha_{13}+\alpha_{14}\right) L_{3}\right)\left(\alpha_{18}+\left(\alpha_{16}+\alpha_{17}\right) L_{1}\right)} \\
& +\frac{\left(\alpha_{11} \alpha_{13}+\alpha_{10} \alpha_{14}\right)\left(\alpha_{16}+\alpha_{17}\right)\left(e^{-L_{1}}+L_{3}\right)\left(e^{-L_{2}}+L_{1}\right)\left(e^{-L_{3}}+L_{2}\right)}{\left(\alpha_{12}+\left(\alpha_{10}+\alpha_{11}\right) L_{2}\right)\left(\alpha_{15}+\left(\alpha_{13}+\alpha_{14}\right) L_{3}\right)\left(\alpha_{18}+\left(\alpha_{16}+\alpha_{17}\right) L_{1}\right)}<1 ;
\end{aligned}
$$

(ii) $\Upsilon_{1}$ is a source if

$$
\begin{aligned}
& \frac{\alpha_{10} \alpha_{13}\left(\alpha_{14}+\alpha_{17}\right)\left(e^{-U_{1}}+U_{3}\right)\left(e^{-U_{2}}+U_{1}\right)\left(e^{-U_{3}}+U_{2}\right)}{\left(\alpha_{12}+\left(\alpha_{10}+\alpha_{11}\right) U_{2}\right)\left(\alpha_{15}+\left(\alpha_{13}+\alpha_{14}\right) U_{3}\right)\left(\alpha_{18}+\left(\alpha_{16}+\alpha_{17}\right) U_{1}\right)} \\
& +\frac{\alpha_{10} \alpha_{14}\left(\alpha_{13}+\alpha_{17}\right)\left(e^{-U_{1}}+U_{3}\right)\left(e^{-U_{2}}+U_{1}\right)\left(e^{-U_{3}}+U_{2}\right)}{\left(\alpha_{12}+\left(\alpha_{10}+\alpha_{11}\right) U_{2}\right)\left(\alpha_{15}+\left(\alpha_{13}+\alpha_{14}\right) U_{3}\right)\left(\alpha_{18}+\left(\alpha_{16}+\alpha_{17}\right) U_{1}\right)} \\
& +\frac{\left(\alpha_{11} \alpha_{13}+\alpha_{10} \alpha_{14}\right)\left(\alpha_{16}+\alpha_{17}\right)\left(e^{-U_{1}}+U_{3}\right)\left(e^{-U_{2}}+U_{1}\right)\left(e^{-U_{3}}+U_{2}\right)}{\left(\alpha_{12}+\left(\alpha_{10}+\alpha_{11}\right) U_{2}\right)\left(\alpha_{15}+\left(\alpha_{13}+\alpha_{14}\right) U_{3}\right)\left(\alpha_{18}+\left(\alpha_{16}+\alpha_{17}\right) U_{1}\right)}>1 .
\end{aligned}
$$

Proof. (i) If $\Upsilon_{1}$ is a fixed point of (3), then

$$
\begin{aligned}
& \bar{x}=\frac{\left(\alpha_{10}+\alpha_{11}\right) e^{-\bar{y}}}{\alpha_{12}+\left(\alpha_{10}+\alpha_{11}\right) \bar{y}}, \\
& \bar{y}=\frac{\left(\alpha_{13}+\alpha_{14}\right) e^{-\bar{z}}}{\alpha_{15}+\left(\alpha_{13}+\alpha_{14}\right) \bar{z}}, \\
& \bar{z}=\frac{\left(\alpha_{16}+\alpha_{17}\right) e^{-\bar{x}}}{\alpha_{18}+\left(\alpha_{16}+\alpha_{17}\right) \bar{x}} .
\end{aligned}
$$

Moreover, we have the following map for constructing the corresponding linearized form of (3):

$$
\left(x_{n+1}, x_{n}, y_{n+1}, y_{n}, z_{n+1}, z_{n}\right) \longrightarrow\left(f, f_{1}, g, g_{1}, h, h_{1}\right) \text {, }
$$

where

$$
\begin{aligned}
f & =\frac{\alpha_{10} e^{-y_{n}}+\alpha_{11} e^{-y_{n-1}}}{\alpha_{12}+\alpha_{10} y_{n}+\alpha_{11} y_{n-1}}, \\
f_{1} & =x_{n}, \\
g & =\frac{\alpha_{13} e^{-z_{n}}+\alpha_{14} e^{-z_{n-1}}}{\alpha_{15}+\alpha_{13} z_{n}+\alpha_{14} z_{n-1}}, \\
g_{1} & =y_{n}, \\
h & =\frac{\alpha_{16} e^{-x_{n}}+\alpha_{17} e^{-x_{n-1}}}{\alpha_{18}+\alpha_{16} x_{n}+\alpha_{17} x_{n-1}}, \\
h_{1} & =z_{n} .
\end{aligned}
$$

The $\left.J\right|_{\Upsilon_{1}}$ about $\Upsilon_{1}$ subject to the map (51) is

$$
\left.J\right|_{\Upsilon_{1}}=\left(\begin{array}{cccccc}
0 & 0 & b_{13} & b_{14} & 0 & 0 \\
1 & 0 & 0 & 0 & 0 & 0 \\
0 & 0 & 0 & 0 & b_{35} & b_{36} \\
0 & 0 & 1 & 0 & 0 & 0 \\
b_{51} & b_{52} & 0 & 0 & 0 & 0 \\
0 & 0 & 0 & 0 & 1 & 0
\end{array}\right),
$$

where

$$
\begin{aligned}
& b_{13}=-\frac{\alpha_{10}\left(e^{-\bar{y}}+\bar{x}\right)}{\alpha_{12}+\left(\alpha_{10}+\alpha_{11}\right) \bar{y}}, \\
& b_{14}=-\frac{\alpha_{11}\left(e^{-\bar{y}}+\bar{x}\right)}{\alpha_{12}+\left(\alpha_{10}+\alpha_{11}\right) \bar{y}},
\end{aligned}
$$

$$
b_{35}=-\frac{\alpha_{13}\left(e^{-\bar{z}}+\bar{y}\right)}{\alpha_{15}+\left(\alpha_{13}+\alpha_{14}\right) \bar{z}}
$$$$
b_{36}=-\frac{\alpha_{14}\left(e^{-\bar{z}}+\bar{y}\right)}{\alpha_{15}+\left(\alpha_{13}+\alpha_{14}\right) \bar{z}}
$$$$
b_{51}=-\frac{\alpha_{16}\left(e^{-\bar{x}}+\bar{z}\right)}{\alpha_{18}+\left(\alpha_{16}+\alpha_{17}\right) \bar{x}}
$$$$
b_{52}=-\frac{\alpha_{17}\left(e^{-\bar{x}}+\bar{z}\right)}{\alpha_{18}+\left(\alpha_{16}+\alpha_{17}\right) \bar{x}} .
$$ 
The auxiliary equation of $\left.J\right|_{\Upsilon_{1}}$ about $\Upsilon_{1}$ is

$$
P(\lambda)=\lambda^{6}+L_{1} \lambda^{3}+L_{2} \lambda^{2}+L_{3} \lambda+L_{4}=0,
$$

where

$$
\begin{aligned}
& L_{1}=-b_{13} b_{35} b_{51}, \\
& L_{2}=-b_{13} b_{35} b_{52}-b_{13} b_{36} b_{51}-b_{14} b_{35} b_{51}, \\
& L_{3}=-b_{13} b_{36} b_{52}-b_{14} b_{35} b_{52}-b_{14} b_{36} b_{51}, \\
& L_{4}=-b_{14} b_{36} b_{52} .
\end{aligned}
$$

Now,

$$
\begin{aligned}
& \sum_{i=1}^{4}\left|L_{i}\right|=\frac{\alpha_{10} \alpha_{13}\left(\alpha_{14}+\alpha_{17}\right)\left(e^{-\bar{x}}+\bar{z}\right)\left(e^{-\bar{y}}+\bar{x}\right)\left(e^{-\bar{z}}+\bar{y}\right)}{\left(\alpha_{12}+\left(\alpha_{10}+\alpha_{11}\right) \bar{y}\right)\left(\alpha_{15}+\left(\alpha_{13}+\alpha_{14}\right) \bar{z}\right)\left(\alpha_{18}+\left(\alpha_{16}+\alpha_{17}\right) \bar{x}\right)} \\
& +\frac{\alpha_{10} \alpha_{14}\left(\alpha_{13}+\alpha_{17}\right)\left(e^{-\bar{x}}+\bar{z}\right)\left(e^{-\bar{y}}+\bar{x}\right)\left(e^{-\bar{z}}+\bar{y}\right)}{\left(\alpha_{12}+\left(\alpha_{10}+\alpha_{11}\right) \bar{y}\right)\left(\alpha_{15}+\left(\alpha_{13}+\alpha_{14}\right) \bar{z}\right)\left(\alpha_{18}+\left(\alpha_{16}+\alpha_{17}\right) \bar{x}\right)} \\
& +\frac{\alpha_{11} \alpha_{13}\left(\alpha_{16}+\alpha_{17}\right)\left(e^{-\bar{x}}+\bar{z}\right)\left(e^{-\bar{y}}+\bar{x}\right)\left(e^{-\bar{z}}+\bar{y}\right)}{\left(\alpha_{12}+\left(\alpha_{10}+\alpha_{11}\right) \bar{y}\right)\left(\alpha_{15}+\left(\alpha_{13}+\alpha_{14}\right) \bar{z}\right)\left(\alpha_{18}+\left(\alpha_{16}+\alpha_{17}\right) \bar{x}\right)} \\
& +\frac{\alpha_{10} \alpha_{14}\left(\alpha_{16}+\alpha_{17}\right)\left(e^{-\bar{x}}+\bar{z}\right)\left(e^{-\bar{y}}+\bar{x}\right)\left(e^{-\bar{z}}+\bar{y}\right)}{\left(\alpha_{12}+\left(\alpha_{10}+\alpha_{11}\right) \bar{y}\right)\left(\alpha_{15}+\left(\alpha_{13}+\alpha_{14}\right) \bar{z}\right)\left(\alpha_{18}+\left(\alpha_{16}+\alpha_{17}\right) \bar{x}\right)} \\
& \leq \frac{\alpha_{10} \alpha_{13}\left(\alpha_{14}+\alpha_{17}\right)\left(e^{-L_{1}}+L_{3}\right)\left(e^{-L_{2}}+L_{1}\right)\left(e^{-L_{3}}+L_{2}\right)}{\left(\alpha_{12}+\left(\alpha_{10}+\alpha_{11}\right) L_{2}\right)\left(\alpha_{15}+\left(\alpha_{13}+\alpha_{14}\right) L_{3}\right)\left(\alpha_{18}+\left(\alpha_{16}+\alpha_{17}\right) L_{1}\right)} \\
& +\frac{\alpha_{10} \alpha_{14}\left(\alpha_{13}+\alpha_{17}\right)\left(e^{-L_{1}}+L_{3}\right)\left(e^{-L_{2}}+L_{1}\right)\left(e^{-L_{3}}+L_{2}\right)}{\left(\alpha_{12}+\left(\alpha_{10}+\alpha_{11}\right) L_{2}\right)\left(\alpha_{15}+\left(\alpha_{13}+\alpha_{14}\right) L_{3}\right)\left(\alpha_{18}+\left(\alpha_{16}+\alpha_{17}\right) L_{1}\right)} \\
& +\frac{\alpha_{11} \alpha_{13}\left(\alpha_{16}+\alpha_{17}\right)\left(e^{-L_{1}}+L_{3}\right)\left(e^{-L_{2}}+L_{1}\right)\left(e^{-L_{3}}+L_{2}\right)}{\left(\alpha_{12}+\left(\alpha_{10}+\alpha_{11}\right) L_{2}\right)\left(\alpha_{15}+\left(\alpha_{13}+\alpha_{14}\right) L_{3}\right)\left(\alpha_{18}+\left(\alpha_{16}+\alpha_{17}\right) L_{1}\right)} \\
& +\frac{\alpha_{10} \alpha_{14}\left(\alpha_{16}+\alpha_{17}\right)\left(e^{-L_{1}}+L_{3}\right)\left(e^{-L_{2}}+L_{1}\right)\left(e^{-L_{3}}+L_{2}\right)}{\left(\alpha_{12}+\left(\alpha_{10}+\alpha_{11}\right) L_{2}\right)\left(\alpha_{15}+\left(\alpha_{13}+\alpha_{14}\right) L_{3}\right)\left(\alpha_{18}+\left(\alpha_{16}+\alpha_{17}\right) L_{1}\right)} \\
& =\frac{\alpha_{10} \alpha_{13}\left(\alpha_{14}+\alpha_{17}\right)\left(e^{-L_{1}}+L_{3}\right)\left(e^{-L_{2}}+L_{1}\right)\left(e^{-L_{3}}+L_{2}\right)}{\left(\alpha_{12}+\left(\alpha_{10}+\alpha_{11}\right) L_{2}\right)\left(\alpha_{15}+\left(\alpha_{13}+\alpha_{14}\right) L_{3}\right)\left(\alpha_{18}+\left(\alpha_{16}+\alpha_{17}\right) L_{1}\right)} \\
& +\frac{\alpha_{10} \alpha_{14}\left(\alpha_{13}+\alpha_{17}\right)\left(e^{-L_{1}}+L_{3}\right)\left(e^{-L_{2}}+L_{1}\right)\left(e^{-L_{3}}+L_{2}\right)}{\left(\alpha_{12}+\left(\alpha_{10}+\alpha_{11}\right) L_{2}\right)\left(\alpha_{15}+\left(\alpha_{13}+\alpha_{14}\right) L_{3}\right)\left(\alpha_{18}+\left(\alpha_{16}+\alpha_{17}\right) L_{1}\right)} \\
& +\frac{\left(\alpha_{11} \alpha_{13}+\alpha_{10} \alpha_{14}\right)\left(\alpha_{16}+\alpha_{17}\right)\left(e^{-L_{1}}+L_{3}\right)\left(e^{-L_{2}}+L_{1}\right)\left(e^{-L_{3}}+L_{2}\right)}{\left(\alpha_{12}+\left(\alpha_{10}+\alpha_{11}\right) L_{2}\right)\left(\alpha_{15}+\left(\alpha_{13}+\alpha_{14}\right) L_{3}\right)\left(\alpha_{18}+\left(\alpha_{16}+\alpha_{17}\right) L_{1}\right)} .
\end{aligned}
$$

Assuming that (48) holds, and then from (57), one gets $\sum_{i=1}^{4}\left|L_{i}\right|<1$. Hence, $\Upsilon_{1}$ of (3) is a sink. (ii) Using similar manipulation as in the proof of (i), one has 


$$
\begin{aligned}
& \sum_{i=1}^{4}\left|L_{i}\right| \geq \frac{\alpha_{10} \alpha_{13}\left(\alpha_{14}+\alpha_{17}\right)\left(e^{-U_{1}}+U_{3}\right)\left(e^{-U_{2}}+U_{1}\right)\left(e^{-U_{3}}+U_{2}\right)}{\left(\alpha_{12}+\left(\alpha_{10}+\alpha_{11}\right) U_{2}\right)\left(\alpha_{15}+\left(\alpha_{13}+\alpha_{14}\right) U_{3}\right)\left(\alpha_{18}+\left(\alpha_{16}+\alpha_{17}\right) U_{1}\right)} \\
& +\frac{\alpha_{10} \alpha_{14}\left(\alpha_{13}+\alpha_{17}\right)\left(e^{-U_{1}}+U_{3}\right)\left(e^{-U_{2}}+U_{1}\right)\left(e^{-U_{3}}+U_{2}\right)}{\left(\alpha_{12}+\left(\alpha_{10}+\alpha_{11}\right) U_{2}\right)\left(\alpha_{15}+\left(\alpha_{13}+\alpha_{14}\right) U_{3}\right)\left(\alpha_{18}+\left(\alpha_{16}+\alpha_{17}\right) U_{1}\right)} \\
& +\frac{\alpha_{11} \alpha_{13}\left(\alpha_{16}+\alpha_{17}\right)\left(e^{-U_{1}}+U_{3}\right)\left(e^{-U_{2}}+U_{1}\right)\left(e^{-U_{3}}+U_{2}\right)}{\left(\alpha_{12}+\left(\alpha_{10}+\alpha_{11}\right) U_{2}\right)\left(\alpha_{15}+\left(\alpha_{13}+\alpha_{14}\right) U_{3}\right)\left(\alpha_{18}+\left(\alpha_{16}+\alpha_{17}\right) U_{1}\right)} \\
& +\frac{\alpha_{10} \alpha_{14}\left(\alpha_{16}+\alpha_{17}\right)\left(e^{-U_{1}}+U_{3}\right)\left(e^{-U_{2}}+U_{1}\right)\left(e^{-U_{3}}+U_{2}\right)}{\left(\alpha_{12}+\left(\alpha_{10}+\alpha_{11}\right) U_{2}\right)\left(\alpha_{15}+\left(\alpha_{13}+\alpha_{14}\right) U_{3}\right)\left(\alpha_{18}+\left(\alpha_{16}+\alpha_{17}\right) U_{1}\right)} \\
& =\frac{\alpha_{10} \alpha_{13}\left(\alpha_{14}+\alpha_{17}\right)\left(e^{-U_{1}}+U_{3}\right)\left(e^{-U_{2}}+U_{1}\right)\left(e^{-U_{3}}+U_{2}\right)}{\left(\alpha_{12}+\left(\alpha_{10}+\alpha_{11}\right) U_{2}\right)\left(\alpha_{15}+\left(\alpha_{13}+\alpha_{14}\right) U_{3}\right)\left(\alpha_{18}+\left(\alpha_{16}+\alpha_{17}\right) U_{1}\right)} \\
& +\frac{\alpha_{10} \alpha_{14}\left(\alpha_{13}+\alpha_{17}\right)\left(e^{-U_{1}}+U_{3}\right)\left(e^{-U_{2}}+U_{1}\right)\left(e^{-U_{3}}+U_{2}\right)}{\left(\alpha_{12}+\left(\alpha_{10}+\alpha_{11}\right) U_{2}\right)\left(\alpha_{15}+\left(\alpha_{13}+\alpha_{14}\right) U_{3}\right)\left(\alpha_{18}+\left(\alpha_{16}+\alpha_{17}\right) U_{1}\right)} \\
& +\frac{\left(\alpha_{11} \alpha_{13}+\alpha_{10} \alpha_{14}\right)\left(\alpha_{16}+\alpha_{17}\right)\left(e^{-U_{1}}+U_{3}\right)\left(e^{-U_{2}}+U_{1}\right)\left(e^{-U_{3}}+U_{2}\right)}{\left(\alpha_{12}+\left(\alpha_{10}+\alpha_{11}\right) U_{2}\right)\left(\alpha_{15}+\left(\alpha_{13}+\alpha_{14}\right) U_{3}\right)\left(\alpha_{18}+\left(\alpha_{16}+\alpha_{17}\right) U_{1}\right)}
\end{aligned}
$$

Assuming that (49) holds, and then from (58), one gets $\sum_{i=1}^{4}\left|L_{i}\right|>1$. Hence, $\Upsilon_{1}$ of (3) is a source.

In similar manner, one can explore the local dynamics about $Y_{i}(i=2, \ldots, 6)$, respectively, of systems (4)-(8), as follows.

\section{Theorem 5.}

(i) For $\Upsilon_{2}$ of (4), the following holds:

(i.1) $\Upsilon_{2}$ is a sink if

$$
\begin{aligned}
& \frac{\alpha_{19} \alpha_{25}\left(\alpha_{22}+\alpha_{23}\right)\left(e^{-L_{4}}+L_{5}\right)\left(e^{-L_{6}}+L_{4}\right)\left(e^{-L_{4}}+L_{6}\right)}{\left(\alpha_{27}+\left(\alpha_{25}+\alpha_{26}\right) L_{5}\right)\left(\alpha_{21}+\left(\alpha_{19}+\alpha_{20}\right) L_{6}\right)\left(\alpha_{24}+\left(\alpha_{22}+\alpha_{23}\right) L_{4}\right)} \\
& +\frac{\alpha_{19} \alpha_{26}\left(\alpha_{22}+\alpha_{23}\right)\left(e^{-L_{4}}+L_{5}\right)\left(e^{-L_{5}}+L_{6}\right)\left(e^{-L_{6}}+L_{4}\right)}{\left(\alpha_{27}+\left(\alpha_{25}+\alpha_{26}\right) L_{5}\right)\left(\alpha_{21}+\left(\alpha_{19}+\alpha_{20}\right) L_{6}\right)\left(\alpha_{24}+\left(\alpha_{22}+\alpha_{23}\right) L_{4}\right)} \\
& +\frac{\left(\alpha_{20} \alpha_{22}+\alpha_{20} \alpha_{23}\right)\left(\alpha_{26}+\alpha_{25}\right)\left(e^{-L_{4}}+L_{5}\right)\left(e^{-L_{5}}+L_{6}\right)\left(e^{-L_{6}}+L_{4}\right)}{\left(\alpha_{27}+\left(\alpha_{25}+\alpha_{26}\right) L_{5}\right)\left(\alpha_{21}+\left(\alpha_{19}+\alpha_{20}\right) L_{6}\right)\left(\alpha_{24}+\left(\alpha_{22}+\alpha_{23}\right) L_{4}\right)}<1 .
\end{aligned}
$$

(i.2) $\Upsilon_{2}$ is a source if

$$
\begin{aligned}
& \frac{\alpha_{19} \alpha_{25}\left(\alpha_{22}+\alpha_{23}\right)\left(e^{-U_{4}}+U_{5}\right)\left(e^{-U_{6}}+U_{4}\right)\left(e^{-U_{4}}+U_{6}\right)}{\left(\alpha_{27}+\left(\alpha_{25}+\alpha_{26}\right) U_{5}\right)\left(\alpha_{21}+\left(\alpha_{19}+\alpha_{20}\right) U_{6}\right)\left(\alpha_{24}+\left(\alpha_{22}+\alpha_{23}\right) U_{4}\right)} \\
& +\frac{\alpha_{19} \alpha_{26}\left(\alpha_{22}+\alpha_{23}\right)\left(e^{-U_{4}}+U_{5}\right)\left(e^{-U_{5}}+U_{6}\right)\left(e^{-U_{6}}+U_{4}\right)}{\left(\alpha_{27}+\left(\alpha_{25}+\alpha_{26}\right) U_{5}\right)\left(\alpha_{21}+\left(\alpha_{19}+\alpha_{20}\right) U_{6}\right)\left(\alpha_{24}+\left(\alpha_{22}+\alpha_{23}\right) U_{4}\right)} \\
& +\frac{\left(\alpha_{20} \alpha_{22}+\alpha_{20} \alpha_{23}\right)\left(\alpha_{26}+\alpha_{25}\right)\left(e^{-U_{4}}+U_{5}\right)\left(e^{-U_{5}}+U_{6}\right)\left(e^{-U_{6}}+U_{4}\right)}{\left(\alpha_{27}+\left(\alpha_{25}+\alpha_{26}\right) U_{5}\right)\left(\alpha_{21}+\left(\alpha_{19}+\alpha_{20}\right) U_{6}\right)\left(\alpha_{24}+\left(\alpha_{22}+\alpha_{23}\right) U_{4}\right)}>1,
\end{aligned}
$$

with

$$
\left.J\right|_{\Upsilon_{2}}=\left(\begin{array}{cccccc}
0 & 0 & 0 & 0 & b_{15} & b_{16} \\
1 & 0 & 0 & 0 & 0 & 0 \\
b_{31} & b_{32} & 0 & 0 & 0 & \\
0 & 0 & 1 & 0 & 0 & 0 \\
0 & 0 & b_{53} & b_{54} & 0 & 0 \\
0 & 0 & 0 & 0 & 1 & 0
\end{array}\right)
$$

where

$$
\begin{aligned}
& b_{15}=-\frac{\alpha_{19}\left(e^{-\bar{x}}+\bar{y}\right)}{\alpha_{21}+\left(\alpha_{19}+\alpha_{20}\right) \bar{x}}, \\
& b_{16}=-\frac{\alpha_{20}\left(e^{-\bar{x}}+\bar{y}\right)}{\alpha_{21}+\left(\alpha_{19}+\alpha_{20}\right) \bar{x}}, \\
& b_{31}=-\frac{\alpha_{22}\left(e^{-\bar{z}}+\bar{x}\right)}{\alpha_{24}+\left(\alpha_{22}+\alpha_{23}\right) \bar{z}}, \\
& b_{32}=-\frac{\alpha_{23}\left(e^{-\bar{z}}+\bar{x}\right)}{\alpha_{24}+\left(\alpha_{22}+\alpha_{23}\right) \bar{z}}, \\
& b_{53}=-\frac{\alpha_{25}\left(e^{-\bar{y}}+\bar{z}\right)}{\alpha_{27}+\left(\alpha_{25}+\alpha_{26}\right) \bar{y}}, \\
& b_{54}=-\frac{\alpha_{26}\left(e^{-\bar{y}}+\bar{z}\right)}{\alpha_{27}+\left(\alpha_{25}+\alpha_{26}\right) \bar{y}} ;
\end{aligned}
$$


(ii) For $\Upsilon_{3}$ of (5), the following holds:

(ii.1) $\Upsilon_{3}$ is a sink if

$$
\begin{aligned}
& \frac{\left(\alpha_{28}+\alpha_{29}\right)\left(e^{-L_{7}}+L_{7}\right)}{\alpha_{30}+\left(\alpha_{28}+\alpha_{29}\right) L_{7}}+\frac{\left(\alpha_{31}+\alpha_{32}\right) e^{-L_{8}}}{\alpha_{33}+\left(\alpha_{31}+\alpha_{32}\right) L_{9}}+\frac{\left(\alpha_{34}+\alpha_{35}\right) e^{-L_{9}}}{\alpha_{36}+\left(\alpha_{34}+\alpha_{35}\right) L_{8}} \\
& +\frac{\left(\alpha_{34} \alpha_{28}+\alpha_{29} \alpha_{34}+\alpha_{35} \alpha_{28}+\alpha_{29} \alpha_{35}+\alpha_{29} \alpha_{32}\right) e^{-L_{7}-L_{9}}}{\left(\alpha_{30}+\left(\alpha_{28}+\alpha_{29}\right) L_{7}\right)\left(\alpha_{36}+\left(\alpha_{34}+\alpha_{35}\right) L_{8}\right)} \\
& +\frac{\left(\alpha_{31} \alpha_{34}+\alpha_{35} \alpha_{31}+\alpha_{32} \alpha_{35}\right) e^{-L_{8}-L_{9}}}{\left(\alpha_{33}+\left(\alpha_{31}+\alpha_{32}\right) L_{9}\right)\left(\alpha_{36}+\left(\alpha_{34}+\alpha_{35}\right) L_{8}\right)} \\
& +\frac{\left(\alpha_{28} \alpha_{34}+\alpha_{29} \alpha_{34}+\alpha_{28} \alpha_{35}+\alpha_{29} \alpha_{32}+\alpha_{29} \alpha_{35}\right) L_{7} e^{-L_{9}}}{\left(\alpha_{30}+\left(\alpha_{28}+\alpha_{29}\right) L_{7}\right)\left(\alpha_{36}+\left(\alpha_{34}+\alpha_{35}\right) L_{8}\right)} \\
& +\frac{\left(\alpha_{31} \alpha_{34}+\alpha_{32} \alpha_{34}+\alpha_{31} \alpha_{35}+\alpha_{32} \alpha_{35}\right) L_{7} L_{8}}{\left(\alpha_{33}+\left(\alpha_{31}+\alpha_{32}\right) L_{9}\right)\left(\alpha_{36}+\left(\alpha_{34}+\alpha_{35}\right) L_{8}\right)} \\
& +\frac{\left(\alpha_{28} \alpha_{31} \alpha_{34}+\alpha_{29} \alpha_{31} \alpha_{34}+\alpha_{28} \alpha_{32} \alpha_{34}+\alpha_{28} \alpha_{35} \alpha_{31}\right) e^{-L_{7}-L_{8}-L_{9}}}{\left(\alpha_{30}+\left(\alpha_{28}+\alpha_{29}\right) L_{7}\right)\left(\alpha_{33}+\left(\alpha_{31}+\alpha_{32}\right) L_{9}\right)\left(\alpha_{36}+\left(\alpha_{34}+\alpha_{35}\right) L_{8}\right)}<1 \\
& +\frac{\left(\alpha_{33}+\left(\alpha_{31}+\alpha_{32}\right) L_{9}\right)\left(\alpha_{36}+\left(\alpha_{34}+\alpha_{35}\right) L_{8}\right)}{\left(\alpha_{30}+\left(\alpha_{28}+\alpha_{29}\right) L_{7}\right)\left(\alpha_{33}+\left(\alpha_{31}+\alpha_{32}\right) L_{9}\right)\left(\alpha_{36}+\left(\alpha_{34}+\alpha_{35}\right) L_{8}\right)} \\
& +\frac{\left(\alpha_{29} \alpha_{32} \alpha_{34}+\alpha_{29} \alpha_{31} \alpha_{35}+\alpha_{28} \alpha_{32} \alpha_{35}+\alpha_{29} \alpha_{32} \alpha_{35}\right) e^{-L_{7}-L_{8}-L_{9}}}{\left(\alpha_{30}+\left(\alpha_{28}+\alpha_{29}\right) L_{7}\right)\left(\alpha_{33}+\left(\alpha_{31}+\alpha_{32}\right) L_{9}\right)\left(\alpha_{36}+\left(\alpha_{34}+\alpha_{35}\right) L_{8}\right)} \\
& +\frac{\left(\alpha_{28} \alpha_{32} \alpha_{34}+\alpha_{29} \alpha_{31} \alpha_{35}+\alpha_{28} \alpha_{32} \alpha_{35}+\alpha_{29} \alpha_{32} \alpha_{35}\right) e^{-L_{7}} L_{8} L_{9}}{\left(\alpha_{30}+\alpha_{29} \alpha_{31} \alpha_{34}+\alpha_{28} \alpha_{32} \alpha_{34}+\alpha_{28} \alpha_{35} \alpha_{31}\right) L_{7} L_{8} L_{9}} \\
& \left.+\left(\alpha_{39}\right) L_{7}\right)\left(\alpha_{33}+\left(\alpha_{31}+\alpha_{32}\right) L_{9}\right)\left(\alpha_{36}+\left(\alpha_{34}+\alpha_{35}\right) L_{8}\right)
\end{aligned}
$$


(ii.2) $\Upsilon_{3}$ is a source if

$$
\begin{aligned}
& \frac{\left(\alpha_{28}+\alpha_{29}\right)\left(e^{-U_{7}}+U_{7}\right)}{\alpha_{30}+\left(\alpha_{28}+\alpha_{29}\right) U_{7}}+\frac{\left(\alpha_{31}+\alpha_{32}\right) e^{-U_{8}}}{\alpha_{33}+\left(\alpha_{31}+\alpha_{32}\right) U_{9}}+\frac{\left(\alpha_{34}+\alpha_{35}\right) e^{-U_{9}}}{\alpha_{36}+\left(\alpha_{34}+\alpha_{35}\right) U_{8}} \\
& +\frac{\left(\alpha_{34} \alpha_{28}+\alpha_{29} \alpha_{34}+\alpha_{35} \alpha_{28}+\alpha_{29} \alpha_{35}+\alpha_{29} \alpha_{32}\right) e^{-U_{7}-U_{9}}}{\left(\alpha_{30}+\left(\alpha_{28}+\alpha_{29}\right) U_{7}\right)\left(\alpha_{36}+\left(\alpha_{34}+\alpha_{35}\right) U_{8}\right)} \\
& +\frac{\left(\alpha_{31} \alpha_{34}+\alpha_{35} \alpha_{31}+\alpha_{32} \alpha_{35}\right) e^{-U_{8}-U_{9}}}{\left(\alpha_{33}+\left(\alpha_{31}+\alpha_{32}\right) U_{9}\right)\left(\alpha_{36}+\left(\alpha_{34}+\alpha_{35}\right) U_{8}\right)} \\
& +\frac{\left(\alpha_{28} \alpha_{34}+\alpha_{29} \alpha_{34}+\alpha_{28} \alpha_{35}+\alpha_{29} \alpha_{32}+\alpha_{29} \alpha_{35}\right) U_{7} e^{-U_{9}}}{\left(\alpha_{30}+\left(\alpha_{28}+\alpha_{29}\right) U_{7}\right)\left(\alpha_{36}+\left(\alpha_{34}+\alpha_{35}\right) U_{8}\right)} \\
& +\frac{\left(\alpha_{31} \alpha_{34}+\alpha_{32} \alpha_{34}+\alpha_{31} \alpha_{35}+\alpha_{32} \alpha_{35}\right) U_{7} U_{8}}{\left(\alpha_{33}+\left(\alpha_{31}+\alpha_{32}\right) U_{9}\right)\left(\alpha_{36}+\left(\alpha_{34}+\alpha_{35}\right) U_{8}\right)} \\
& +\frac{\left(\alpha_{28} \alpha_{31} \alpha_{34}+\alpha_{29} \alpha_{31} \alpha_{34}+\alpha_{28} \alpha_{32} \alpha_{34}+\alpha_{28} \alpha_{35} \alpha_{31}\right) e^{-U_{7}-U_{8}-U_{9}}}{\left(\alpha_{30}+\left(\alpha_{28}+\alpha_{29}\right) U_{7}\right)\left(\alpha_{33}+\left(\alpha_{31}+\alpha_{32}\right) U_{9}\right)\left(\alpha_{36}+\left(\alpha_{34}+\alpha_{35}\right) U_{8}\right)} \\
& +\frac{\left.\left(\alpha_{28}+\alpha_{29}\right) U_{7}\right)\left(\alpha_{33}+\left(\alpha_{31}+\alpha_{32}\right) U_{9}\right)\left(\alpha_{36}+\left(\alpha_{34}+\alpha_{35}\right) U_{8}\right)}{\left(\alpha_{30}+\left(\alpha_{28}+\alpha_{29}\right) U_{7}\right)\left(\alpha_{33}+\left(\alpha_{31}+\alpha_{32}\right) U_{9}\right)\left(\alpha_{36}+\left(\alpha_{34}+\alpha_{35}\right) U_{8}\right)} \\
& +
\end{aligned}
$$


with

$$
\left.J\right|_{\Upsilon_{3}}=\left(\begin{array}{cccccc}
b_{11} & b_{12} & 0 & 0 & 0 & 0 \\
1 & 0 & 0 & 0 & 0 & 0 \\
0 & 0 & b_{33} & b_{34} & b_{35} & b_{36} \\
0 & 0 & 1 & 0 & 0 & 0 \\
0 & 0 & b_{53} & b_{54} & b_{55} & b_{56} \\
0 & 0 & 0 & 0 & 1 & 0
\end{array}\right),
$$

where

$$
\begin{aligned}
& b_{11}=-\frac{\alpha_{28}\left(e^{-\bar{x}}+\bar{x}\right)}{\alpha_{30}+\left(\alpha_{28}+\alpha_{29}\right) \bar{x}}, \\
& b_{12}=-\frac{\alpha_{29}\left(e^{-\bar{x}}+\bar{x}\right)}{\alpha_{30}+\left(\alpha_{28}+\alpha_{29}\right) \bar{x}}, \\
& b_{33}=-\frac{\alpha_{31} e^{-\bar{y}}}{\alpha_{33}+\left(\alpha_{31}+\alpha_{32}\right) \bar{z}}, \\
& b_{34}=-\frac{\alpha_{32} e^{-\bar{y}}}{\alpha_{33}+\left(\alpha_{31}+\alpha_{32}\right) \bar{z}}, \\
& b_{35}=-\frac{\alpha_{31} \bar{y}}{\alpha_{33}+\left(\alpha_{31}+\alpha_{32}\right) \bar{z}}, \\
& b_{36}=-\frac{\alpha_{32} \bar{y}}{\alpha_{33}+\left(\alpha_{31}+\alpha_{32}\right) \bar{z}}, \\
& b_{53}=-\frac{\alpha_{34} \bar{z}}{\alpha_{36}+\left(\alpha_{34}+\alpha_{35}\right) \bar{y}}, \\
& b_{54}=-\frac{\alpha_{35} \bar{z}}{\alpha_{36}+\left(\alpha_{34}+\alpha_{35}\right) \bar{y}}, \\
& b_{55}=-\frac{\alpha_{34} e^{-\bar{z}}}{\alpha_{36}+\left(\alpha_{34}+\alpha_{35}\right) \bar{y}}, \\
& \alpha_{36}+\left(\alpha_{34}+\alpha_{35}\right) \bar{y}
\end{aligned}
$$

$$
\begin{aligned}
& \frac{\left(\alpha_{37}+\alpha_{38}\right) e^{-L_{10}}}{\alpha_{39}+\left(\alpha_{37}+\alpha_{38}\right) L_{11}}+\frac{\left(\alpha_{40}+\alpha_{41}\right) e^{-L_{11}}}{\alpha_{42}+\left(\alpha_{40}+\alpha_{41}\right) L_{10}} \\
& +\frac{\left(\alpha_{43}+\alpha_{44}\right)\left(e^{-L_{12}}+L_{12}\right)}{\alpha_{45}+\left(\alpha_{43}+\alpha_{44}\right) L_{12}} \\
& +\frac{\left(\alpha_{37} \alpha_{40}+\alpha_{41} \alpha_{37}+\alpha_{38} \alpha_{40}+\alpha_{37} \alpha_{41}+\alpha_{38} \alpha_{41}\right) e^{-L_{10}-L_{11}}}{\left(\alpha_{39}+\left(\alpha_{37}+\alpha_{38}\right) L_{11}\right)\left(\alpha_{42}+\left(\alpha_{40}+\alpha_{41}\right) L_{10}\right)} \\
& +\frac{\left(\alpha_{37} \alpha_{40}+\alpha_{38} \alpha_{40}+\alpha_{37} \alpha_{41}+\alpha_{38} \alpha_{41}\right) L_{10} L_{11}}{\left(\alpha_{39}+\left(\alpha_{37}+\alpha_{38}\right) L_{11}\right)\left(\alpha_{42}+\left(\alpha_{40}+\alpha_{41}\right) L_{10}\right)} \\
& +\frac{\left(\alpha_{40} \alpha_{43}+\alpha_{41} \alpha_{44}+\alpha_{41} \alpha_{43}+\alpha_{4} \alpha_{44}\right) e^{-L_{11}}\left(e^{-L_{12}}+L_{12}\right)}{\left(\alpha_{42}+\left(\alpha_{40}+\alpha_{41}\right) L_{10}\right)\left(\alpha_{45}+\left(\alpha_{43}+\alpha_{44}\right) L_{12}\right)} \\
& +\frac{\left(\alpha_{37} \alpha_{43}+\alpha_{38} \alpha_{43}+\alpha_{38} \alpha_{44}+\alpha_{37} \alpha_{44}\right) e^{-L_{10}}\left(e^{-L_{12}}+L_{10}\right)}{\left(\alpha_{39}+\left(\alpha_{37}+\alpha_{38}\right) L_{11}\right)\left(\alpha_{45}+\left(\alpha_{43}+\alpha_{44}\right) L_{12}\right)} \\
& +\left[\left(\alpha_{37} \alpha_{40} \alpha_{43}+\alpha_{38} \alpha_{40} \alpha_{43}+\alpha_{37} \alpha_{41} \alpha_{43}+\alpha_{37} \alpha_{40} \alpha_{44}\right.\right. \\
& \left.+\alpha_{38} \alpha_{41} \alpha_{43}+\alpha_{38} \alpha_{40} \alpha_{44}+\alpha_{37} \alpha_{41} \alpha_{44}+\alpha_{38} \alpha_{41} \alpha_{44}\right) e^{-L_{10}-L_{11}-L_{12}} \\
& +\left(\alpha_{37} \alpha_{40} \alpha_{43}+\alpha_{38} \alpha_{40} \alpha_{43}+\alpha_{37} \alpha_{41} \alpha_{43}+\alpha_{37} \alpha_{40} \alpha_{44}\right. \\
& \left.+\alpha_{38} \alpha_{41} \alpha_{43}+\alpha_{38} \alpha_{40} \alpha_{44}+\alpha_{37} \alpha_{41} \alpha_{44}+\alpha_{38} \alpha_{41} \alpha_{44}\right) L_{10} L_{11} L_{12} \\
& +\left(\alpha_{37} \alpha_{40} \alpha_{43}+\alpha_{38} \alpha_{40} \alpha_{43}+\alpha_{37} \alpha_{41} \alpha_{43}+\alpha_{37} \alpha_{40} \alpha_{44}\right. \\
& \left.+\alpha_{38} \alpha_{41} \alpha_{43}+\alpha_{38} \alpha_{40} \alpha_{44}+\alpha_{37} \alpha_{41} \alpha_{44}+\alpha_{38} \alpha_{41} \alpha_{44}\right) e^{-L_{12}} L_{10} L_{11} \\
& +\left(\alpha_{37} \alpha_{40} \alpha_{43}+\alpha_{38} \alpha_{40} \alpha_{43}+\alpha_{37} \alpha_{41} \alpha_{43}+\alpha_{37} \alpha_{40} \alpha_{44}+\alpha_{38} \alpha_{41} \alpha_{43}\right. \\
& \left.\left.+\alpha_{38} \alpha_{40} \alpha_{44}+\alpha_{37} \alpha_{41} \alpha_{44}+\alpha_{38} \alpha_{41} \alpha_{44}\right) L_{12} e^{-L_{10}-L_{11}}\right] \\
& \frac{1}{\left(\alpha_{39}+\left(\alpha_{37}+\alpha_{38}\right) L_{11}\right)\left(\alpha_{42}+\left(\alpha_{40}+\alpha_{41}\right) L_{10}\right)\left(\alpha_{45}+\left(\alpha_{43}+\alpha_{44}\right) L_{12}\right)} \\
& <1
\end{aligned}
$$

(iii) For $\Upsilon_{4}$ of (6), the following holds:

(iii.1) $\Upsilon_{4}$ is a sink if (iii.2) $\Upsilon_{4}$ is a source if 


$$
\begin{aligned}
& \frac{\left(\alpha_{37}+\alpha_{38}\right) e^{-U_{10}}}{\alpha_{39}+\left(\alpha_{37}+\alpha_{38}\right) U_{11}}+\frac{\left(\alpha_{40}+\alpha_{41}\right) e^{-U_{11}}}{\alpha_{42}+\left(\alpha_{40}+\alpha_{41}\right) U_{10}} \\
& +\frac{\left(\alpha_{43}+\alpha_{44}\right)\left(e^{-U_{12}}+L_{12}\right)}{\alpha_{45}+\left(\alpha_{43}+\alpha_{44}\right) U_{12}} \\
& +\frac{\left(\alpha_{37} \alpha_{40}+\alpha_{41} \alpha_{37}+\alpha_{38} \alpha_{40}+\alpha_{37} \alpha_{41}+\alpha_{38} \alpha_{41}\right) e^{-U_{10}-U_{11}}}{\left(\alpha_{39}+\left(\alpha_{37}+\alpha_{38}\right) U_{11}\right)\left(\alpha_{42}+\left(\alpha_{40}+\alpha_{41}\right) U_{10}\right)} \\
& +\frac{\left(\alpha_{37} \alpha_{40}+\alpha_{38} \alpha_{40}+\alpha_{37} \alpha_{41}+\alpha_{38} \alpha_{41}\right) U_{10} U_{11}}{\left(\alpha_{39}+\left(\alpha_{37}+\alpha_{38}\right) U_{11}\right)\left(\alpha_{42}+\left(\alpha_{40}+\alpha_{41}\right) U_{10}\right)} \\
& +\frac{\left(\alpha_{40} \alpha_{43}+\alpha_{41} \alpha_{44}+\alpha_{41} \alpha_{43}+\alpha_{4} \alpha_{44}\right) e^{-U_{11}}\left(e^{-U_{12}}+U_{12}\right)}{\left(\alpha_{42}+\left(\alpha_{40}+\alpha_{41}\right) U_{10}\right)\left(\alpha_{45}+\left(\alpha_{43}+\alpha_{44}\right) U_{12}\right)} \\
& +\frac{\left(\alpha_{37} \alpha_{43}+\alpha_{38} \alpha_{43}+\alpha_{38} \alpha_{44}+\alpha_{37} \alpha_{44}\right) e^{-U_{10}}\left(e^{-U_{12}}+U_{10}\right)}{\left(\alpha_{39}+\left(\alpha_{37}+\alpha_{38}\right) U_{11}\right)\left(\alpha_{45}+\left(\alpha_{43}+\alpha_{44}\right) U_{12}\right)} \\
& +\left[\left(\alpha_{37} \alpha_{40} \alpha_{43}+\alpha_{38} \alpha_{40} \alpha_{43}+\alpha_{37} \alpha_{41} \alpha_{43}+\alpha_{37} \alpha_{40} \alpha_{44}+\right.\right. \\
& \left.\alpha_{38} \alpha_{41} \alpha_{43}+\alpha_{38} \alpha_{40} \alpha_{44}+\alpha_{37} \alpha_{41} \alpha_{44}+\alpha_{38} \alpha_{41} \alpha_{44}\right) e^{-U_{10}-U_{11}-U_{12}} \\
& +\left(\alpha_{37} \alpha_{40} \alpha_{43}+\alpha_{38} \alpha_{40} \alpha_{43}+\alpha_{37} \alpha_{41} \alpha_{43}+\alpha_{37} \alpha_{40} \alpha_{44}\right. \\
& \left.+\alpha_{38} \alpha_{41} \alpha_{43}+\alpha_{38} \alpha_{40} \alpha_{44}+\alpha_{37} \alpha_{41} \alpha_{44}+\alpha_{38} \alpha_{41} \alpha_{44}\right) U_{10} U_{11} U_{12} \\
& +\left(\alpha_{37} \alpha_{40} \alpha_{43}+\alpha_{38} \alpha_{40} \alpha_{43}+\alpha_{37} \alpha_{41} \alpha_{43}+\alpha_{37} \alpha_{40} \alpha_{44}\right. \\
& \left.+\alpha_{38} \alpha_{41} \alpha_{43}+\alpha_{38} \alpha_{40} \alpha_{44}+\alpha_{37} \alpha_{41} \alpha_{44}+\alpha_{38} \alpha_{41} \alpha_{44}\right) e^{-U_{12}} U_{10} U_{11} \\
& +\left(\alpha_{37} \alpha_{40} \alpha_{43}+\alpha_{38} \alpha_{40} \alpha_{43}+\alpha_{37} \alpha_{41} \alpha_{43}+\alpha_{37} \alpha_{40} \alpha_{44}+\alpha_{38} \alpha_{41} \alpha_{43}\right. \\
& \left.\left.+\alpha_{38} \alpha_{40} \alpha_{44}+\alpha_{37} \alpha_{41} \alpha_{44}+\alpha_{38} \alpha_{41} \alpha_{44}\right) U_{12} e^{-U_{10}-U_{11}}\right] \\
& \times \frac{1}{\left(\alpha_{39}+\left(\alpha_{37}+\alpha_{38}\right) U_{11}\right)\left(\alpha_{42}+\left(\alpha_{40}+\alpha_{41}\right) U_{10}\right)\left(\alpha_{45}+\left(\alpha_{43}+\alpha_{44}\right) U_{12}\right)} \\
& >1 \text {, }
\end{aligned}
$$

with

$$
\left.J\right|_{\Upsilon_{4}}=\left(\begin{array}{cccccc}
b_{11} & b_{12} & b_{13} & b_{14} & 0 & 0 \\
1 & 0 & 0 & 0 & 0 & 0 \\
b_{31} & b_{32} & b_{33} & b_{34} & 0 & 0 \\
0 & 0 & 1 & 0 & 0 & 0 \\
0 & 0 & 0 & 0 & b_{55} & b_{56} \\
0 & 0 & 0 & 0 & 1 & 0
\end{array}\right),
$$

where

$$
\begin{aligned}
& b_{11}=-\frac{\alpha_{37} e^{-\bar{x}}}{\alpha_{39}+\left(\alpha_{37}+\alpha_{38}\right) \bar{y}} \\
& b_{12}=-\frac{\alpha_{38} e^{-\bar{x}}}{\alpha_{39}+\left(\alpha_{37}+\alpha_{38}\right) \bar{y}} \\
& b_{13}=-\frac{\alpha_{37} \bar{x}}{\alpha_{39}+\left(\alpha_{37}+\alpha_{38}\right) \bar{y}} \\
& b_{14}=-\frac{\alpha_{38} \bar{x}}{\alpha_{39}+\left(\alpha_{37}+\alpha_{38}\right) \bar{y}} \\
& b_{31}=-\frac{\alpha_{40} \bar{y}}{\alpha_{42}+\left(\alpha_{40}+\alpha_{41}\right) \bar{x}} \\
& b_{32}=-\frac{\alpha_{41} \bar{y}}{\alpha_{42}+\left(\alpha_{40}+\alpha_{41}\right) \bar{x}} \\
& b_{56}=-\frac{\alpha_{44}\left(e^{-\bar{z}}+\bar{z}\right)}{\alpha_{45}+\left(\alpha_{43}+\alpha_{44}\right) \bar{z}} \\
& b_{53}=-\frac{\alpha_{40} e^{-\bar{y}}}{\alpha_{45}+\left(\alpha_{43}+\alpha_{44}\right) \bar{z}} \\
& b_{34}=-\frac{\alpha_{41} e^{-\bar{y}}}{\alpha_{42}+\left(\alpha_{40}+\alpha_{41}\right) \bar{x}}
\end{aligned}
$$

(iv) For $\Upsilon_{5}$ of (7), the following holds: (iv.1) $\Upsilon_{5}$ is a sink if 


$$
\begin{aligned}
& \frac{\left(\alpha_{46}+\alpha_{47}\right) e^{-L_{13}}}{\alpha_{48}+\left(\alpha_{46}+\alpha_{47}\right) L_{15}}+\frac{\left(\alpha_{52}+\alpha_{53}\right) e^{-L_{15}}}{\alpha_{54}+\left(\alpha_{52}+\alpha_{53}\right) L_{13}}+\frac{\left(\alpha_{49}+\alpha_{50}\right)\left(e^{-L_{14}}+L_{14}\right)}{\alpha_{51}+\left(\alpha_{49}+\alpha_{50}\right) L_{14}} \\
& +\frac{\left(\alpha_{46} \alpha_{52}+\alpha_{47} \alpha_{52}+\alpha_{46} \alpha_{53}+\alpha_{47} \alpha_{53}\right) e^{-L_{13}-L_{15}}}{\left(\alpha_{48}+\left(\alpha_{46}+\alpha_{47}\right) L_{15}\right)\left(\alpha_{54}+\left(\alpha_{52}+\alpha_{53}\right) L_{13}\right)} \\
& +\frac{\left(\alpha_{46} \alpha_{52}+\alpha_{47} \alpha_{52}+\alpha_{46} \alpha_{53}+\alpha_{47} \alpha_{53}\right) L_{13} L_{15}}{\left(\alpha_{48}+\left(\alpha_{46}+\alpha_{47}\right) L_{15}\right)\left(\alpha_{54}+\left(\alpha_{52}+\alpha_{53}\right) L_{13}\right)} \\
& +\frac{\left(\alpha_{\alpha_{46}} \alpha_{49}+\alpha_{47} \alpha_{47}+\alpha_{46} \alpha_{50}+\alpha_{47} \alpha_{50}\right) e^{-L_{13}}\left(e^{-L_{14}}+L_{14}\right)}{\left(\alpha_{48}+\left(\alpha_{46}+\alpha_{47}\right) L_{15}\right)\left(\alpha_{51}+\left(\alpha_{49}+\alpha_{50}\right) L_{14}\right)} \\
& +\frac{\left(\alpha_{49} \alpha_{52}+\alpha_{50} \alpha_{52}+\alpha_{49} \alpha_{53}+\alpha_{50} \alpha_{53}\right) e^{-L_{15}}\left(e^{-L_{14}}+L_{14}\right)}{\left(\alpha_{54}+\left(\alpha_{52}+\alpha_{53}\right) L_{13}\right)\left(\alpha_{51}+\left(\alpha_{49}+\alpha_{50}\right) L_{14}\right)} \\
& +\left[\left(\alpha_{46} \alpha_{49} \alpha_{52}+\alpha_{47} \alpha_{49} \alpha_{52}+\alpha_{46} \alpha_{50} \alpha_{52}+\alpha_{46} \alpha_{49} \alpha_{53}\right.\right. \\
& \left.+\alpha_{47} \alpha_{50} \alpha_{52}+\alpha_{47} \alpha_{49} \alpha_{53}+\alpha_{46} \alpha_{50} \alpha_{53}+\alpha_{47} \alpha_{50} \alpha_{53}\right) e^{-L_{13}-L_{14}-L_{15}} \\
& +\left(\alpha_{46} \alpha_{49} \alpha_{52}+\alpha_{47} \alpha_{49} \alpha_{52}+\alpha_{46} \alpha_{50} \alpha_{52}+\alpha_{46} \alpha_{49} \alpha_{53}\right. \\
& \left.+\alpha_{47} \alpha_{50} \alpha_{52}+\alpha_{47} \alpha_{49} \alpha_{53}+\alpha_{46} \alpha_{50} \alpha_{53}+\alpha_{47} \alpha_{50} \alpha_{53}\right) L_{13} L_{14} L_{15} \\
& +\left(\alpha_{46} \alpha_{49} \alpha_{52}+\alpha_{47} \alpha_{49} \alpha_{52}+\alpha_{46} \alpha_{50} \alpha_{52}+\alpha_{46} \alpha_{49} \alpha_{53}\right. \\
& \left.+\alpha_{47} \alpha_{50} \alpha_{52}+\alpha_{47} \alpha_{49} \alpha_{53}+\alpha_{46} \alpha_{50} \alpha_{53}+\alpha_{47} \alpha_{50} \alpha_{53}\right) e^{-L_{14} L_{13} L_{15}} \\
& +\left(\alpha_{46} \alpha_{49} \alpha_{52}+\alpha_{47} \alpha_{49} \alpha_{52}+\alpha_{46} \alpha_{50} \alpha_{52}+\alpha_{46} \alpha_{49} \alpha_{53}\right. \\
& \left.\left.+\alpha_{47} \alpha_{50} \alpha_{52}+\alpha_{47} \alpha_{49} \alpha_{53}+\alpha_{46} \alpha_{50} \alpha_{53}+\alpha_{47} \alpha_{50} \alpha_{53}\right) L_{14} e^{-L_{13}-L_{15}}\right] \\
& \times \frac{1}{\left(\alpha_{48}+\left(\alpha_{46}+\alpha_{47}\right) L_{15}\right)\left(\alpha_{51}+\left(\alpha_{49}+\alpha_{50}\right) L_{14}\right)\left(\alpha_{54}+\left(\alpha_{52}+\alpha_{53}\right) L_{13}\right)}<1 .
\end{aligned}
$$


(iv.2) $\Upsilon_{5}$ is a source if

$$
\begin{gathered}
\frac{\left(\alpha_{46}+\alpha_{47}\right) e^{-U_{13}}}{\alpha_{48}+\left(\alpha_{46}+\alpha_{47}\right) U_{15}}+\frac{\left(\alpha_{52}+\alpha_{53}\right) e^{-U_{15}}}{\alpha_{54}+\left(\alpha_{52}+\alpha_{53}\right) U_{13}} \\
+\frac{\left(\alpha_{49}+\alpha_{50}\right)\left(e^{-U_{14}}+U_{14}\right)}{\alpha_{51}+\left(\alpha_{49}+\alpha_{50}\right) U_{14}}+
\end{gathered}
$$

$\frac{\left(\alpha_{46} \alpha_{52}+\alpha_{47} \alpha_{52}+\alpha_{46} \alpha_{53}+\alpha_{47} \alpha_{53}\right) e^{-U_{13}-U_{15}}}{\left(\alpha_{54}+\left(\alpha_{52}+\alpha_{53}\right) U_{13}\right)\left(\alpha_{48}+\left(\alpha_{46}+\alpha_{47}\right) U_{15}\right)}$

$\left(\alpha_{54}+\left(\alpha_{52}+\alpha_{53}\right) U_{13}\right)\left(\alpha_{48}+\left(\alpha_{46}+\alpha_{47}\right) U_{15}\right)$

$+\frac{\left(\alpha_{46} \alpha_{52}+\alpha_{47} \alpha_{52}+\alpha_{46} \alpha_{53}+\alpha_{47} \alpha_{53}\right) U_{13} U_{15}}{\left(\alpha_{54}+\left(\alpha_{52}+\alpha_{53}\right) U_{13}\right)\left(\alpha_{48}+\left(\alpha_{46}+\alpha_{47}\right) U_{15}\right)}$

$+\frac{\left(\alpha_{\alpha_{46}} \alpha_{49}+\alpha_{47} \alpha_{47}+\alpha_{46} \alpha_{50}+\alpha_{47} \alpha_{50}\right) e^{-U_{13}}\left(e^{-U_{14}}+U_{14}\right)}{\left(\alpha_{54}+\left(\alpha_{52}+\alpha_{53}\right) U_{15}\right)\left(\alpha_{51}+\left(\alpha_{49}+\alpha_{48}\right) U_{14}\right)}$

$+\frac{\left(\alpha_{49} \alpha_{52}+\alpha_{50} \alpha_{52}+\alpha_{49} \alpha_{53}+\alpha_{50} \alpha_{53}\right) e^{-U_{15}}\left(e^{-U_{14}}+U_{14}\right)}{\left(\alpha_{54}+\left(\alpha_{52}+\alpha_{53}\right) U_{15}\right)\left(\alpha_{51}+\left(\alpha_{49}+\alpha_{48}\right) U_{14}\right)}$

$+\left[\left(\alpha_{46} \alpha_{49} \alpha_{52}+\alpha_{47} \alpha_{49} \alpha_{52}+\alpha_{46} \alpha_{50} \alpha_{52}+\alpha_{46} \alpha_{49} \alpha_{53}+\right.\right.$

$\left.\alpha_{47} \alpha_{50} \alpha_{52}+\alpha_{47} \alpha_{49} \alpha_{53}+\alpha_{46} \alpha_{50} \alpha_{53}+\alpha_{47} \alpha_{50} \alpha_{53}\right) e^{-U_{13}-U_{14}-U_{15}}$

$+\left(\alpha_{46} \alpha_{49} \alpha_{52}+\alpha_{47} \alpha_{49} \alpha_{52}+\alpha_{46} \alpha_{50} \alpha_{52}+\alpha_{46} \alpha_{49} \alpha_{53}\right.$

$\left.+\alpha_{47} \alpha_{50} \alpha_{52}+\alpha_{47} \alpha_{49} \alpha_{53}+\alpha_{46} \alpha_{50} \alpha_{53}+\alpha_{47} \alpha_{50} \alpha_{53}\right) U_{13} U_{14} U_{15}$

$+\left(\alpha_{46} \alpha_{49} \alpha_{52}+\alpha_{47} \alpha_{49} \alpha_{52}+\alpha_{46} \alpha_{50} \alpha_{52}+\alpha_{46} \alpha_{49} \alpha_{53}\right.$

$\left.+\alpha_{47} \alpha_{50} \alpha_{52}+\alpha_{47} \alpha_{49} \alpha_{53}+\alpha_{46} \alpha_{50} \alpha_{53}+\alpha_{47} \alpha_{50} \alpha_{53}\right) e^{-U_{14}} U_{13} U_{15}$

$+\left(\alpha_{46} \alpha_{49} \alpha_{52}+\alpha_{47} \alpha_{49} \alpha_{52}+\alpha_{46} \alpha_{50} \alpha_{52}+\alpha_{46} \alpha_{49} \alpha_{53}+\right.$

$\left.\left.\alpha_{47} \alpha_{50} \alpha_{52}+\alpha_{47} \alpha_{49} \alpha_{53}+\alpha_{46} \alpha_{50} \alpha_{53}+\alpha_{47} \alpha_{50} \alpha_{53}\right) U_{14} e^{-U_{13}-U_{15}}\right]$

$\times \frac{1}{\left(\alpha_{48}+\left(\alpha_{46}+\alpha_{47}\right) U_{15}\right)\left(\alpha_{51}+\left(\alpha_{49}+\alpha_{50}\right) U_{14}\right)\left(\alpha_{54}\left(\alpha_{52}+\alpha_{53}\right) U_{13}\right)}$

$>1$,

with

$$
\left.J\right|_{\Upsilon_{5}}=\left(\begin{array}{cccccc}
b_{11} & b_{12} & 0 & 0 & b_{15} & b_{16} \\
1 & 0 & 0 & 0 & 0 & 0 \\
0 & 0 & b_{33} & b_{34} & 0 & 0 \\
0 & 0 & 1 & 0 & 0 & 0 \\
b_{51} & b_{52} & 0 & 0 & b_{55} & b_{56} \\
0 & 0 & 0 & 0 & 1 & 0
\end{array}\right) \text {, }
$$

where

$$
\begin{aligned}
& b_{11}=-\frac{\alpha_{46} e^{-\bar{x}}}{\alpha_{48}+\left(\alpha_{46}+\alpha_{47}\right) \bar{z}}, \\
& b_{12}=-\frac{\alpha_{47} e^{-\bar{x}}}{\alpha_{48}+\left(\alpha_{46}+\alpha_{47}\right) \bar{z}}, \\
& b_{15}=-\frac{\alpha_{46} \bar{x}}{\alpha_{48}+\left(\alpha_{46}+\alpha_{47}\right) \bar{z}}, \\
& b_{16}=-\frac{\alpha_{47} \bar{x}}{\alpha_{48}+\left(\alpha_{46}+\alpha_{47}\right) \bar{z}}, \\
& b_{33}=-\frac{\alpha_{49}\left(e^{-\bar{y}}+\bar{y}\right)}{\alpha_{51}+\left(\alpha_{49}+\alpha_{50}\right) \bar{y}}, \\
& b_{34}=-\frac{\alpha_{50}\left(e^{-\bar{y}}+\bar{y}\right)}{\alpha_{51}+\left(\alpha_{49}+\alpha_{50}\right) \bar{y}},
\end{aligned}
$$

$$
b_{51}=-\frac{\alpha_{52} \bar{z}}{\alpha_{54}+\left(\alpha_{52}+\alpha_{53}\right) \bar{x}}
$$$$
b_{52}=-\frac{\alpha_{53} \bar{z}}{\alpha_{54}+\left(\alpha_{52}+\alpha_{53}\right) \bar{x}}
$$$$
b_{55}=-\frac{\alpha_{52} e^{-\bar{z}}}{\alpha_{54}+\left(\alpha_{52}+\alpha_{53}\right) \bar{x}}
$$$$
b_{56}=-\frac{\alpha_{53} e^{-\bar{z}}}{\alpha_{54}+\left(\alpha_{52}+\alpha_{53}\right) \bar{x}}
$$

(v) For $\Upsilon_{6}$ of (8), the following holds:

(v.1) $\Upsilon_{6}$ is a sink if 


$$
\begin{aligned}
& \frac{\left(\alpha_{55} \alpha_{58}+\alpha_{56} \alpha_{58}+\alpha_{55} \alpha_{59}+\alpha_{56} \alpha_{59}\right) L_{16} L_{17}}{\left(\alpha_{57}+\left(\alpha_{55}+\alpha_{56}\right) L_{16}\right)\left(\alpha_{60}+\left(\alpha_{58}+\alpha_{59}\right) L_{17}\right)}+\frac{\left(\alpha_{55}+\alpha_{56}\right) L_{16}}{\alpha_{57}+\left(\alpha_{55}+\alpha_{56}\right) L_{16}} \\
& +\frac{\left(\alpha_{55} \alpha_{61}+\alpha_{55} \alpha_{62}+\alpha_{56} \alpha_{61}+\alpha_{56} \alpha_{62}\right) L_{16} L_{18}}{\left(\alpha_{57}+\left(\alpha_{55}+\alpha_{56}\right) L_{16}\right)\left(\alpha_{63}+\left(\alpha_{61}+\alpha_{62}\right) L_{18}\right)}+\frac{\left(\alpha_{58}+\alpha_{59}\right) L_{17}}{\alpha_{60}+\left(\alpha_{58}+\alpha_{59}\right) L_{17}} \\
& +\frac{\left(\alpha_{58} \alpha_{62}+\alpha_{59} \alpha_{61}+\alpha_{59} \alpha_{62}+\alpha_{58} \alpha_{61}\right) L_{16} L_{18}}{\left(\alpha_{57}+\left(\alpha_{55}+\alpha_{56}\right) L_{16}\right)\left(\alpha_{63}+\left(\alpha_{61}+\alpha_{62}\right) L_{18}\right)}+\frac{\left(\alpha_{61}+\alpha_{62}\right) L_{18}}{\alpha_{63}+\left(\alpha_{61}+\alpha_{62}\right) L_{18}} \\
& +\left[\left(\alpha_{55} \alpha_{58} \alpha_{61}+\alpha_{56} \alpha_{58} \alpha_{61}+\alpha_{55} \alpha_{58} \alpha_{61}+\alpha_{55} \alpha_{58} \alpha_{62}\right.\right. \\
& \left.+\alpha_{56} \alpha_{58} \alpha_{61}+\alpha_{56} \alpha_{58} \alpha_{62}+\alpha_{55} \alpha_{58} \alpha_{62}+\alpha_{56} \alpha_{58} \alpha_{62}\right) e^{-L_{16}-L_{17}-L_{18}} \\
& +\left(\alpha_{55} \alpha_{58} \alpha_{61}+\alpha_{55} \alpha_{58} \alpha_{62}+\alpha_{56} \alpha_{58} \alpha_{61}+\alpha_{55} \alpha_{59} \alpha_{61}\right. \\
& \left.\left.+\alpha_{56} \alpha_{58} \alpha_{62}+\alpha_{55} \alpha_{59} \alpha_{62}+\alpha_{56} \alpha_{59} \alpha_{61}+\alpha_{56} \alpha_{59} \alpha_{62}\right) L_{16} L_{17} L_{18}\right] \\
& \times \frac{1}{\left(\alpha_{57}+\left(\alpha_{55}+\alpha_{56}\right) L_{16}\right)\left(\alpha_{60}+\left(\alpha_{58}+\alpha_{59}\right) L_{17}\right)\left(\alpha_{63}+\left(\alpha_{61}+\alpha_{62}\right) L_{18}\right)}<1
\end{aligned}
$$

(v.2) $\Upsilon_{6}$ is a source if

$$
\begin{aligned}
& \frac{\left(\alpha_{55} \alpha_{58}+\alpha_{56} \alpha_{58}+\alpha_{55} \alpha_{59}+\alpha_{56} \alpha_{59}\right) U_{16} U_{17}}{\left(\alpha_{57}+\left(\alpha_{55}+\alpha_{56}\right) U_{16}\right)\left(\alpha_{60}+\left(\alpha_{58}+\alpha_{59}\right) U_{17}\right)}+\frac{\left(\alpha_{55}+\alpha_{56}\right) U_{16}}{\alpha_{57}+\left(\alpha_{55}+\alpha_{56}\right) U_{16}} \\
& +\frac{\left(\alpha_{55} \alpha_{61}+\alpha_{55} \alpha_{62}+\alpha_{56} \alpha_{61}+\alpha_{56} \alpha_{62}\right) U_{16} U_{18}}{\left(\alpha_{57}+\left(\alpha_{55}+\alpha_{56}\right) U_{16}\right)\left(\alpha_{63}+\left(\alpha_{61}+\alpha_{62}\right) U_{18}\right)}+\frac{\left(\alpha_{58}+\alpha_{59}\right) U_{17}}{\alpha_{60}+\left(\alpha_{58}+\alpha_{59}\right) U_{17}} \\
& +\frac{\left(\alpha_{58} \alpha_{62}+\alpha_{59} \alpha_{61}+\alpha_{59} \alpha_{62}+\alpha_{58} \alpha_{61}\right) U_{16} U_{18}}{\left(\alpha_{57}+\left(\alpha_{55}+\alpha_{56}\right) U_{16}\right)\left(\alpha_{63}+\left(\alpha_{61}+\alpha_{62}\right) U_{18}\right)}+\frac{\left(\alpha_{61}+\alpha_{62}\right) U_{18}}{\alpha_{63}+\left(\alpha_{61}+\alpha_{62}\right) U_{18}} \\
& +\left[\left(\alpha_{55} \alpha_{58} \alpha_{61}+\alpha_{56} \alpha_{58} \alpha_{61}+\alpha_{55} \alpha_{58} \alpha_{61}+\alpha_{55} \alpha_{58} \alpha_{62}\right.\right. \\
& \left.+\alpha_{56} \alpha_{58} \alpha_{61}+\alpha_{56} \alpha_{58} \alpha_{62}+\alpha_{55} \alpha_{58} \alpha_{62}+\alpha_{56} \alpha_{58} \alpha_{62}\right) e^{-U_{16}-U_{17}-U_{18}} \\
& +\left(\alpha_{55} \alpha_{58} \alpha_{61}+\alpha_{55} \alpha_{58} \alpha_{62}+\alpha_{56} \alpha_{58} \alpha_{61}+\alpha_{55} \alpha_{59} \alpha_{61}\right. \\
& \left.\left.+\alpha_{56} \alpha_{58} \alpha_{62}+\alpha_{55} \alpha_{59} \alpha_{62}+\alpha_{56} \alpha_{59} \alpha_{61}+\alpha_{56} \alpha_{59} \alpha_{62}\right) U_{16} U_{17} U_{18}\right] \\
& \quad \times \frac{1}{\left(\alpha_{57}+\left(\alpha_{55}+\alpha_{56}\right) U_{16}\right)\left(\alpha_{60}+\left(\alpha_{58}+\alpha_{59}\right) U_{17}\right)\left(\alpha_{63}+\left(\alpha_{61}+\alpha_{62}\right) U_{18}\right)}>1
\end{aligned}
$$

with 


$$
\left.J\right|_{Y_{6}}=\left(\begin{array}{cccccc}
b_{11} & b_{12} & 0 & 0 & b_{15} & b_{16} \\
1 & 0 & 0 & 0 & 0 & 0 \\
b_{31} & b_{32} & b_{33} & b_{34} & 0 & 0 \\
0 & 0 & 1 & 0 & 0 & 0 \\
0 & 0 & b_{53} & b_{54} & b_{55} & b_{56} \\
0 & 0 & 0 & 0 & 1 & 0
\end{array}\right),
$$

where

$$
\begin{aligned}
& b_{11}=-\frac{\alpha_{55} \bar{x}}{\alpha_{57}+\left(\alpha_{55}+\alpha_{56}\right) \bar{x}}, \\
& b_{12}=-\frac{\alpha_{56} \bar{x}}{\alpha_{57}+\left(\alpha_{55}+\alpha_{56}\right) \bar{x}}, \\
& b_{15}=-\frac{\alpha_{55} e^{-\bar{z}}}{\alpha_{57}+\left(\alpha_{55}+\alpha_{56}\right) \bar{x}}, \\
& b_{16}=-\frac{\alpha_{56} e^{-\bar{z}}}{\alpha_{57}+\left(\alpha_{55}+\alpha_{56}\right) \bar{x}}, \\
& b_{31}=-\frac{\alpha_{58} e^{-\bar{x}}}{\alpha_{60}+\left(\alpha_{58}+\alpha_{59}\right) \bar{y}} \\
& b_{32}=-\frac{\alpha_{59} e^{-\bar{x}}}{\alpha_{60}+\left(\alpha_{58}+\alpha_{59}\right) \bar{y}}, \\
& b_{33}=-\frac{\alpha_{58} \bar{y}}{\alpha_{60}+\left(\alpha_{58}+\alpha_{59}\right) \bar{y}}, \\
& b_{34}=-\frac{\alpha_{59} \bar{y}}{\alpha_{60}+\left(\alpha_{58}+\alpha_{59}\right) \bar{y}}, \\
& b_{53}=-\frac{\alpha_{61} e^{-\bar{y}}}{\alpha_{63}+\left(\alpha_{61}+\alpha_{62}\right) \bar{z}}, \\
& b_{54}=-\frac{\alpha_{62} e^{-\bar{y}}}{\alpha_{63}+\left(\alpha_{61}+\alpha_{62}\right) \bar{z}} \\
& b_{55}=-\frac{\alpha_{61} \bar{z}}{\alpha_{63}+\left(\alpha_{61}+\alpha_{62}\right) \bar{z}}, \\
& b_{56}=-\frac{\alpha_{62} \bar{z}}{\alpha_{63}+\left(\alpha_{61}+\alpha_{62}\right) \bar{z}} .
\end{aligned}
$$

Proof. It is similar to the proof of Theorem 4. So its proof is omitted.

Hereafter, by constructing a Lyapunov function with discrete time motivated from the work of [2], global dynamics about $Y_{i}(i=1, \ldots, 6)$, respectively, of systems (3)-(8) is explored.

\section{Global Dynamics of Systems (3)-(8)}

Theorem 6. For global dynamics about $Y_{i}(i=1, \ldots, 6)$, respectively, of systems (3)-(8), following statements hold:

(i) $\Upsilon_{1}$ of (3) is global asymptotically stable if

$$
\begin{aligned}
& \left(\alpha_{10}+\alpha_{11}\right) e^{-L_{2}}<\left(2 \bar{x}-U_{1}\right)\left(\alpha_{12}+\left(\alpha_{10}+\alpha_{11}\right) L_{2}\right), \\
& \left(\alpha_{13}+\alpha_{14}\right) e^{-L_{3}}<\left(2 \bar{y}-U_{2}\right)\left(\alpha_{15}+\left(\alpha_{13}+\alpha_{14}\right) L_{3}\right), \\
& \left(\alpha_{16}+\alpha_{17}\right) e^{-L_{1}}<\left(2 \bar{z}-U_{3}\right)\left(\alpha_{18}+\left(\alpha_{16}+\alpha_{17}\right) L_{1}\right) ;
\end{aligned}
$$

(ii) $\Upsilon_{2}$ of (4) is global asymptotically stable if

$$
\begin{aligned}
& \left(\alpha_{19}+\alpha_{20}\right) e^{-L_{6}}<\left(2 \bar{x}-U_{4}\right)\left(\alpha_{21}+\left(\alpha_{19}+\alpha_{20}\right) L_{6}\right), \\
& \left(\alpha_{22}+\alpha_{23}\right) e^{-L_{4}}<\left(2 \bar{y}-U_{5}\right)\left(\alpha_{24}+\left(\alpha_{22}+\alpha_{23}\right) L_{4}\right), \\
& \left(\alpha_{25}+\alpha_{26}\right) e^{-L_{5}}<\left(2 \bar{z}-U_{6}\right)\left(\alpha_{27}+\left(\alpha_{25}+\alpha_{26}\right) L_{5}\right)
\end{aligned}
$$

(iii) $\Upsilon_{3}$ of (5) is global asymptotically stable if

$$
\begin{aligned}
& \left(\alpha_{28}+\alpha_{29}\right) e^{-L_{7}}<\left(2 \bar{x}-U_{7}\right)\left(\alpha_{30}+\left(\alpha_{28}+\alpha_{29}\right) L_{7}\right), \\
& \left(\alpha_{31}+\alpha_{32}\right) e^{-L_{8}}<\left(2 \bar{y}-U_{8}\right)\left(\alpha_{33}+\left(\alpha_{31}+\alpha_{32}\right) L_{9}\right), \\
& \left(\alpha_{34}+\alpha_{35}\right) e^{-L_{9}}<\left(2 \bar{z}-U_{9}\right)\left(\alpha_{36}+\left(\alpha_{34}+\alpha_{35}\right) L_{8}\right) ;
\end{aligned}
$$

(iv) $\Upsilon_{4}$ of (6) is global asymptotically stable if

$$
\begin{aligned}
& \left(\alpha_{37}+\alpha_{38}\right) e^{-L_{10}}<\left(2 \bar{x}-U_{10}\right)\left(\alpha_{39}+\left(\alpha_{37}+\alpha_{38}\right) L_{11}\right) \\
& \left(\alpha_{40}+\alpha_{41}\right) e^{-L_{11}}<\left(2 \bar{y}-U_{11}\right)\left(\alpha_{42}+\left(\alpha_{40}+\alpha_{41}\right) L_{10}\right) \\
& \left(\alpha_{43}+\alpha_{44}\right) e^{-L_{12}}<\left(2 \bar{z}-U_{12}\right)\left(\alpha_{45}+\left(\alpha_{43}+\alpha_{44}\right) L_{12}\right)
\end{aligned}
$$

(v) $\Upsilon_{5}$ of (7) is global asymptotically stable if

$$
\begin{aligned}
& \left(\alpha_{46}+\alpha_{47}\right) e^{-L_{13}}<\left(2 \bar{x}-U_{13}\right)\left(\alpha_{48}+\left(\alpha_{46}+\alpha_{47}\right) L_{15}\right), \\
& \left(\alpha_{49}+\alpha_{50}\right) e^{-L_{14}}<\left(2 \bar{y}-U_{14}\right)\left(\alpha_{51}+\left(\alpha_{49}+\alpha_{50}\right) L_{14}\right) \\
& \left(\alpha_{52}+\alpha_{53}\right) e^{-L_{15}}<\left(2 \bar{z}-U_{15}\right)\left(\alpha_{54}+\left(\alpha_{52}+\alpha_{53}\right) L_{13}\right)
\end{aligned}
$$

(vi) $\Upsilon_{6}$ of (8) is global asymptotically stable if

$$
\begin{aligned}
& \left(\alpha_{55}+\alpha_{56}\right) e^{-L_{18}}<\left(2 \bar{x}-U_{16}\right)\left(\alpha_{57}+\left(\alpha_{56}+\alpha_{57}\right) L_{16}\right), \\
& \left(\alpha_{58}+\alpha_{59}\right) e^{-L_{16}}<\left(2 \bar{y}-U_{17}\right)\left(\alpha_{60}+\left(\alpha_{58}+\alpha_{59}\right) L_{17}\right), \\
& \left(\alpha_{61}+\alpha_{62}\right) e^{-L_{17}}<\left(2 \bar{z}-U_{18}\right)\left(\alpha_{63}+\left(\alpha_{61}+\alpha_{62}\right) L_{18}\right) .
\end{aligned}
$$

Proof. (i) Consider the following discrete-time Lyapunov function: 


$$
\Gamma_{n}=\left(x_{n}-\bar{x}\right)^{2}+\left(y_{n}-\bar{y}\right)^{2}+\left(z_{n}-\bar{z}\right)^{2} .
$$

$$
\begin{aligned}
& \Delta \Gamma_{n}=\Gamma_{n+1}-\Gamma_{n} \\
& =\left(x_{n+1}-x_{n}\right)\left(x_{n+1}+x_{n}-2 \bar{x}\right)+\left(y_{n+1}-y_{n}\right)\left(y_{n+1}+y_{n}-2 \bar{y}\right)+\left(z_{n+1}-z_{n}\right)\left(z_{n+1}+z_{n}-2 \bar{z}\right), \\
& =\left(x_{n+1}-x_{n}\right)\left(\frac{\alpha_{10} e^{-y_{n}}+\alpha_{11} e^{-y_{n-1}}}{\alpha_{12}+\alpha_{10} y_{n}+\alpha_{11} y_{n-1}}+x_{n}-2 \bar{x}\right) \\
& +\left(y_{n+1}-y_{n}\right)\left(\frac{\alpha_{13} e^{-z_{n}}+\alpha_{14} e^{-z_{n-1}}}{\alpha_{15}+\alpha_{13} z_{n}+\alpha_{14} z_{n-1}}+y_{n}-2 \bar{y}\right) \\
& +\left(z_{n+1}-z_{n}\right)\left(\frac{\alpha_{16} e^{-x_{n}}+\alpha_{17} e^{-x_{n-1}}}{\alpha_{18}+\alpha_{16} x_{n}+\alpha_{17} x_{n-1}}+z_{n}-2 \bar{z}\right) \\
& \leq\left(U_{1}-L_{1}\right)\left(\frac{\left(\alpha_{10}+\alpha_{11}\right) e^{-L_{2}}}{\alpha_{12}+\left(\alpha_{10}+\alpha_{11}\right) L_{2}}+U_{1}-2 \bar{x}\right) \\
& +\left(U_{2}-L_{2}\right)\left(\frac{\left(\alpha_{13}+\alpha_{14}\right) e^{-L_{3}}}{\alpha_{15}+\left(\alpha_{13}+\alpha_{14}\right) L_{3}}+U_{2}-2 \bar{y}\right) \\
& +\left(U_{3}-L_{3}\right)\left(\frac{\left(\alpha_{16}+\alpha_{17}\right) e^{-L_{1}}}{\alpha_{18}+\left(\alpha_{16}+\alpha_{17}\right) L_{1}}+U_{3}-2 \bar{z}\right) \\
& =\left(U_{1}-L_{1}\right)\left(\frac{\left(\alpha_{10}+\alpha_{11}\right) e^{-L_{2}}-\left(2 \bar{x}-U_{1}\right)\left(\alpha_{12}+\left(\alpha_{10}+\alpha_{11}\right) L_{2}\right)}{\alpha_{12}+\left(\alpha_{10}+\alpha_{11}\right) L_{2}}\right) \\
& +\left(U_{2}-L_{2}\right)\left(\frac{\left(\alpha_{13}+\alpha_{14}\right) e^{-L_{3}}-\left(2 \bar{y}-U_{2}\right)\left(\alpha_{15}+\left(\alpha_{13}+\alpha_{14}\right) L_{3}\right)}{\alpha_{15}+\left(\alpha_{13}+\alpha_{14}\right) L_{3}}\right) \\
& +\left(U_{3}-L_{3}\right)\left(\frac{\left(\alpha_{16}+\alpha_{17}\right) e^{-L_{1}}-\left(2 \bar{z}-U_{3}\right)\left(\alpha_{18}+\left(\alpha_{16}+\alpha_{17}\right) L_{1}\right)}{\alpha_{18}+\left(\alpha_{16}+\alpha_{17}\right) L_{1}}\right) .
\end{aligned}
$$

From (80) and (87), one gets $\Delta \Gamma_{n}<0 \forall n \geq 0$. Hence, we obtain that $\lim _{n \longrightarrow \infty}\left(\Gamma_{n+1}-\Gamma_{n}\right)=0$, and thus $\Upsilon_{1}$ of (3) is global asymptotically stable.

Remark 3. The proof of (ii)-(vi) is same as the proof of (i).

\section{Rate of Convergence}

We will explore the convergence result about the equilibrium point of systems (3)-(8) motivated from the existing literature [3-5], in this section.

Theorem 7. If the positive solution of (3) is $\left\{\Omega_{n}\right\}_{n=-1}^{\infty}$, s.t.

$$
\begin{aligned}
& \lim _{n \longrightarrow \infty} x_{n}=\bar{x}, \\
& \lim _{n \longrightarrow \infty} y_{n}=\bar{y}, \\
& \lim _{n \longrightarrow \infty} z_{n}=\bar{z},
\end{aligned}
$$

where

$$
\begin{aligned}
& \bar{x} \in\left[L_{1}, U_{1}\right], \\
& \bar{y} \in\left[L_{2}, U_{2}\right], \\
& \bar{z} \in\left[L_{3}, U_{3}\right],
\end{aligned}
$$

then the error vector, i.e.,

$$
\theta_{n}=\left(\begin{array}{c}
\theta_{n}^{1} \\
\theta_{n-1}^{1} \\
\theta_{n}^{2} \\
\theta_{n-1}^{2} \\
\theta_{n}^{3} \\
\theta_{n-1}^{3}
\end{array}\right),
$$

satisfies the following relation: 


$$
\begin{gathered}
\lim _{n \longrightarrow \infty}\left(\left\|\theta_{n}\right\|\right)^{1 / n}=\mid \lambda_{1, \ldots, 6} J_{\left|\Upsilon_{1}\right|}, \\
\lim _{n \longrightarrow \infty} \frac{\left\|\theta_{n+1}\right\|}{\left\|\theta_{n}\right\|}=\mid \lambda_{1, \ldots, 6} J_{\left|\Upsilon_{1}\right|},
\end{gathered}
$$

where $\left|\lambda_{1, \ldots, 6} J\right|_{\Upsilon_{1} \mid}$ are the roots of $\left.J\right|_{\Upsilon_{1}}$.

(90)

Proof. If the positive solution of (3) is $\left\{\Omega_{n}\right\}_{n=-1}^{\infty}$, s.t. (88) along with (89) holds. To find the error terms, one has

$$
\begin{aligned}
& x_{n+1}-\bar{x}=\frac{\alpha_{10} e^{-y_{n}}+\alpha_{11} e^{-y_{n-1}}}{\alpha_{12}+\alpha_{10} y_{n}+\alpha_{11} y_{n-1}}-\frac{\left(\alpha_{10}+\alpha_{11}\right) e^{-\bar{y}}}{\alpha_{12}+\left(\alpha_{10}+\alpha_{11}\right) \bar{y}} \\
& =-\frac{\alpha_{10} e^{-y_{n}}\left(e^{y_{n}-\bar{y}}-1\right)}{\alpha_{12}+\alpha_{10} y_{n}+\alpha_{11} y_{n-1}}-\frac{\alpha_{11} e^{-y_{n-1}}\left(e^{y_{n-1}-\bar{y}}-1\right)}{\alpha_{12}+\alpha_{10} y_{n}+\alpha_{11} y_{n-1}}-\frac{\alpha_{10} \bar{x}\left(y_{n}-\bar{y}\right)}{\alpha_{12}+\alpha_{10} y_{n}+\alpha_{11} y_{n-1}} \\
& -\frac{\alpha_{11} \bar{x}\left(y_{n-1}-\bar{y}\right)}{\alpha_{12}+\alpha_{10} y_{n}+\alpha_{11} y_{n-1}} \\
& =-\frac{\alpha_{10}\left(e^{-y_{n}}\left(y_{n}-\bar{y}+O_{1}\left(\left(y_{n}-\bar{y}\right)^{2}\right)\right)+\bar{x}\left(y_{n}-\bar{y}\right)\right)}{\alpha_{12}+\alpha_{10} y_{n}+\alpha_{11} y_{n-1}}\left(y_{n}-\bar{y}\right) \\
& -\frac{\alpha_{11}\left(e^{-y_{n-1}}\left(y_{n-1}-\bar{y}+O_{2}\left(\left(y_{n-1}-\bar{y}\right)^{2}\right)+\bar{x}\left(y_{n-1}-\bar{y}\right)\right)\right.}{\alpha_{12}+\alpha_{10} y_{n}+\alpha_{11} y_{n-1}}\left(y_{n-1}-\bar{y}\right) .
\end{aligned}
$$

So,

$$
\begin{aligned}
x_{n+1}-\bar{x}= & -\frac{\alpha_{10}\left(e^{-y_{n}}+\bar{x}\right)}{\alpha_{12}+\alpha_{10} y_{n}+\alpha_{11} y_{n-1}}\left(y_{n}-\bar{y}\right)-\frac{\alpha_{11}\left(e^{-y_{n-1}}+\bar{x}\right)}{\alpha_{12}+\alpha_{10} y_{n}+\alpha_{11} y_{n-1}}\left(y_{n-1}-\bar{y}\right) \\
& +O_{1}\left(\left(y_{n}-\bar{y}\right)^{2}\right)+O_{2}\left(\left(y_{n-1}-\bar{y}\right)^{2}\right) .
\end{aligned}
$$

Similarly,

$$
\begin{aligned}
y_{n+1}-\bar{y}= & -\frac{\alpha_{13}\left(e^{-z_{n}}+\bar{y}\right)}{\alpha_{15}+\alpha_{13} z_{n}+\alpha_{14} z_{n-1}}\left(z_{n}-\bar{z}\right)-\frac{\alpha_{14}\left(e^{\left.-z_{n-1}+\bar{y}\right)}\right.}{\alpha_{15}+\alpha_{13} z_{n}+\alpha_{14} z_{n-1}}\left(z_{n-1}-\bar{z}\right) \\
& +O_{3}\left(\left(z_{n}-\bar{z}\right)^{2}\right)+O_{4}\left(\left(z_{n-1}-\bar{z}\right)^{2}\right), \\
z_{n+1}-\bar{z}= & -\frac{\alpha_{16}\left(e^{-x_{n}}+\bar{z}\right)}{\alpha_{18}+\alpha_{16} x_{n}+\alpha_{17} x_{n-1}}\left(x_{n}-\bar{x}\right)-\frac{\alpha_{17}\left(e^{\left.-x_{n-1}+\bar{z}\right)}\right.}{\alpha_{18}+\alpha_{16} x_{n}+\alpha_{17} x_{n-1}}\left(x_{n-1}-\bar{x}\right)+ \\
& O_{5}\left(\left(x_{n}-\bar{x}\right)^{2}\right)+O_{6}\left(\left(x_{n-1}-\bar{x}\right)^{2}\right) .
\end{aligned}
$$

From (92) and (93), one gets

$$
\begin{aligned}
& x_{n+1}-\bar{x} \approx-\frac{\alpha_{10}\left(e^{-y_{n}}+\bar{x}\right)}{\alpha_{12}+\alpha_{10} y_{n}+\alpha_{11} y_{n-1}}\left(y_{n}-\bar{y}\right)-\frac{\alpha_{11}\left(e^{-y_{n-1}}+\bar{x}\right)}{\alpha_{12}+\alpha_{10} y_{n}+\alpha_{11} y_{n-1}}\left(y_{n-1}-\bar{y}\right), \\
& y_{n+1}-\bar{y} \approx-\frac{\alpha_{13}\left(e^{-z_{n}}+\bar{y}\right)}{\alpha_{15}+\alpha_{13} z_{n}+\alpha_{14} z_{n-1}}\left(z_{n}-\bar{z}\right)-\frac{\alpha_{14}\left(e^{-z_{n-1}}+\bar{y}\right)}{\alpha_{15}+\alpha_{13} z_{n}+\alpha_{14} z_{n-1}}\left(z_{n-1}-\bar{z}\right), \\
& z_{n+1}-\bar{z} \approx-\frac{\alpha_{16}\left(e^{-x_{n}}+\bar{z}\right)}{\alpha_{18}+\alpha_{16} x_{n}+\alpha_{17} x_{n-1}}\left(x_{n}-\bar{x}\right)-\frac{\alpha_{17}\left(e^{-x_{n-1}}+\bar{z}\right)}{\alpha_{18}+\alpha_{16} x_{n}+\alpha_{17} x_{n-1}}\left(x_{n-1}-\bar{x}\right) .
\end{aligned}
$$


Denote

$$
\begin{aligned}
& \theta_{n}^{1}=x_{n}-\bar{x} \\
& \theta_{n}^{2}=y_{n}-\bar{y} \\
& \theta_{n}^{3}=z_{n}-\bar{z} .
\end{aligned}
$$

In view of (95), from (94), one gets

$$
\begin{aligned}
& \theta_{n+1}^{1}=\omega_{1 n} \theta_{n}^{2}+\omega_{2 n} \theta_{n-1}^{2}, \\
& \theta_{n+1}^{2}=\omega_{3 n} \theta_{n}^{3}+\omega_{4 n} \theta_{n-1}^{3}, \\
& \theta_{n+1}^{3}=\omega_{5 n} \theta_{n}^{1}+\omega_{6 n} \theta_{n-1}^{1},
\end{aligned}
$$

where

$$
\begin{aligned}
& \omega_{1 n}=-\frac{\alpha_{10}\left(e^{-y_{n}}+\bar{x}\right)}{\alpha_{12}+\alpha_{10} y_{n}+\alpha_{11} y_{n-1}}, \\
& \omega_{2 n}=-\frac{\alpha_{11}\left(e^{-y_{n-1}}+\bar{x}\right)}{\alpha_{12}+\alpha_{10} y_{n}+\alpha_{11} y_{n-1}}, \\
& \omega_{3 n}=-\frac{\alpha_{13}\left(e^{-z_{n}}+\bar{y}\right)}{\alpha_{15}+\alpha_{13} z_{n}+\alpha_{14} z_{n-1}}, \\
& \omega_{4 n}=-\frac{\alpha_{14}\left(e^{-z_{n-1}}+\bar{y}\right)}{\alpha_{15}+\alpha_{13} z_{n}+\alpha_{14} z_{n-1}}, \\
& \omega_{5 n}=-\frac{\alpha_{16}\left(e^{-x_{n}}+\bar{z}\right)}{\alpha_{18}+\alpha_{16} x_{n}+\alpha_{17} x_{n-1}}, \\
& \omega_{6 n}=-\frac{\alpha_{17}\left(e^{-x_{n-1}}+\bar{z}\right)}{\alpha_{18}+\alpha_{16} x_{n}+\alpha_{17} x_{n-1}} .
\end{aligned}
$$

From (97), one gets

$$
\begin{aligned}
& \lim _{n \longrightarrow \infty} \omega_{1 n}=-\frac{\alpha_{10}\left(e^{-\bar{y}}+\bar{x}\right)}{\alpha_{12}+\left(\alpha_{10}+\alpha_{11}\right) \bar{y}} \\
& \lim _{n \longrightarrow \infty} \omega_{2 n}=-\frac{\alpha_{11}\left(e^{-\bar{y}}+\bar{x}\right)}{\alpha_{12}+\left(\alpha_{10}+\alpha_{11}\right) \bar{y}} \\
& \lim _{n \longrightarrow \infty} \omega_{3 n}=-\frac{\alpha_{13}\left(e^{-\bar{z}}+\bar{y}\right)}{\alpha_{15}+\left(\alpha_{13}+\alpha_{14}\right) \bar{z}} \\
& \lim _{n \longrightarrow \infty} \omega_{4 n}=-\frac{\alpha_{14}\left(e^{-\bar{z}}+\bar{y}\right)}{\alpha_{15}+\left(\alpha_{13}+\alpha_{14}\right) \bar{z}} \\
& \lim _{n \longrightarrow \infty} \omega_{5 n}=-\frac{\alpha_{16}\left(e^{-\bar{x}}+\bar{z}\right)}{\alpha_{18}+\left(\alpha_{16}+\alpha_{17}\right) \bar{x}} \\
& \lim _{n \longrightarrow \infty} \omega_{6 n}=-\frac{\alpha_{17}\left(e^{-\bar{x}}+\bar{z}\right)}{\alpha_{18}+\left(\alpha_{16}+\alpha_{17}\right) \bar{x}}
\end{aligned}
$$

that is,

$$
\begin{aligned}
& \omega_{1 n}=-\frac{\alpha_{10}\left(e^{-\bar{y}}+\bar{x}\right)}{\alpha_{12}+\left(\alpha_{10}+\alpha_{11}\right) \bar{y}}+b 1_{n}, \\
& \omega_{2 n}=-\frac{\alpha_{11}\left(e^{-\bar{y}}+\bar{x}\right)}{\alpha_{12}+\left(\alpha_{10}+\alpha_{11}\right) \bar{y}}+b 1_{n-1},
\end{aligned}
$$

$$
\begin{aligned}
& \omega_{3 n}=-\frac{\alpha_{13}\left(e^{-\bar{z}}+\bar{y}\right)}{\alpha_{15}+\left(\alpha_{13}+\alpha_{14}\right) \bar{z}}+b 2_{n}, \\
& \omega_{4 n}=-\frac{\alpha_{14}\left(e^{-\bar{z}}+\bar{y}\right)}{\alpha_{15}+\left(\alpha_{13}+\alpha_{14}\right) \bar{z}}+b 2_{n-1},
\end{aligned}
$$

$$
\begin{aligned}
& \omega_{5 n}=-\frac{\alpha_{16}\left(e^{-\bar{x}}+\bar{z}\right)}{\alpha_{18}+\left(\alpha_{16}+\alpha_{17}\right) \bar{x}}+b 3_{n}, \\
& \omega_{6 n}=-\frac{\alpha_{17}\left(e^{-\bar{x}}+\bar{z}\right)}{\alpha_{18}+\left(\alpha_{16}+\alpha_{17}\right) \bar{x}}+b 3_{n-1},
\end{aligned}
$$

where $\quad b 1_{n}, b 1_{n-1}, b 2_{n}, b 2_{n-1}, b 3_{n}$, and $b 3_{n-1} \longrightarrow 0 \quad$ as $n \longrightarrow \infty$. Now, we have system 1.10 of [6], where $A=\left.J\right|_{\Upsilon_{1}}$ and

$$
B(n)=\left(\begin{array}{cccccc}
0 & 0 & b 1_{n} & b 1_{n-1} & 0 & 0 \\
1 & 0 & 0 & 0 & 0 & 0 \\
0 & 0 & 0 & 0 & b 2_{n} & b 2_{n-1} \\
0 & 0 & 1 & 0 & 0 & 0 \\
b 3_{n} & b 3_{n-1} & 0 & 0 & 0 & 0 \\
0 & 0 & 0 & 0 & 1 & 0
\end{array}\right) \text {, }
$$

such that $\|B(n)\| \longrightarrow \infty, n \longrightarrow \infty$. So, about $\Upsilon_{1}$ of $(3)$ the limiting system becomes

$$
\left(\begin{array}{c}
\theta_{n+1}^{1} \\
\theta_{n}^{1} \\
\theta_{n+1}^{2} \\
\theta_{n}^{2} \\
\theta_{n+1}^{3} \\
\theta_{n}^{3}
\end{array}\right)=\left.J\right|_{\Upsilon_{1}}\left(\begin{array}{c}
\theta_{n}^{1} \\
\theta_{n-1}^{1} \\
\theta_{n}^{2} \\
\theta_{n-1}^{2} \\
\theta_{n}^{3} \\
\theta_{n-1}^{3}
\end{array}\right)
$$

which is as $\left.J\right|_{\Upsilon_{1}}$ about $\Upsilon_{1}$.

In the following theorem, we will summarize the convergence results for systems (4) to (8).

Theorem 8. (i) If the positive solution of (4) is $\left\{\Omega_{n}\right\}_{n=-1}^{\infty}$, s.t. (87) along with the following relation holds:

$$
\begin{aligned}
& \bar{x} \in\left[L_{4}, U_{4}\right], \\
& \bar{y} \in\left[L_{5}, U_{5}\right], \\
& \bar{z} \in\left[L_{6}, U_{6}\right],
\end{aligned}
$$


then the error vector, which is depicted in (89), satisfies the following relations:

$$
\begin{gathered}
\lim _{n \longrightarrow \infty}\left(\left\|\theta_{n}\right\|\right)^{1 / n}=\left|\lambda_{1, \ldots, 6} J\right| \Upsilon_{2} \mid, \\
\lim _{n \longrightarrow \infty} \frac{\left\|\theta_{n+1}\right\|}{\left\|\theta_{n}\right\|}=\left|\lambda_{1, \ldots, 6} J\right| \Upsilon_{2} \mid,
\end{gathered}
$$

where $\left|\lambda_{1, \ldots, 6} J\right| \Upsilon_{2} \mid$ are the roots of $J \mid \Upsilon_{2}$.

(ii) If the positive solution of (5) is $\left\{\Omega_{n}\right\}_{n=-1}^{\infty}$, s.t. (87) along with the following relation holds:

$$
\begin{aligned}
& \bar{x} \in\left[L_{7}, U_{7}\right], \\
& \bar{y} \in\left[L_{8}, U_{8}\right], \\
& \bar{z} \in\left[L_{9}, U_{9}\right],
\end{aligned}
$$

then the error vector, which is depicted in (89), satisfies the following relations:

$$
\begin{array}{r}
\lim _{n \longrightarrow \infty}\left(\left\|\theta_{n}\right\|\right)^{1 / n}=\left|\lambda_{1, \ldots, 6} J\right| \Upsilon_{3} \mid, \\
\lim _{n \longrightarrow \infty} \frac{\left\|\theta_{n+1}\right\|}{\left\|\theta_{n}\right\|}=\left|\lambda_{1, \ldots, 6} J\right| \Upsilon_{3} \mid,
\end{array}
$$

where $\left|\lambda_{1, \ldots, 6} J\right| \Upsilon_{3} \mid$ are the roots of $J \mid \Upsilon_{3}$.

(iii) If the positive solution of (6) is $\left\{\Omega_{n}\right\}_{n=-1}^{\infty}$, s.t. (87) along with the following relation holds:

$$
\begin{aligned}
& \bar{x} \in\left[L_{10}, U_{10}\right], \\
& \bar{y} \in\left[L_{11}, U_{11}\right], \\
& \bar{z} \in\left[L_{12}, U_{12}\right],
\end{aligned}
$$

then the error vector, which is depicted in (89), satisfies the following relations:

$$
\begin{gathered}
\lim _{n \longrightarrow \infty}\left(\left\|\theta_{n}\right\|\right)^{1 / n}=\left|\lambda_{1, \ldots, 6} J\right| \Upsilon_{4} \mid, \\
\lim _{n \longrightarrow \infty} \frac{\left\|\theta_{n+1}\right\|}{\left\|\theta_{n}\right\|}=\left|\lambda_{1, \ldots, 6} J\right| \Upsilon_{4} \mid,
\end{gathered}
$$

where $\left|\lambda_{1, \ldots, 6} J\right| \Upsilon_{4} \mid$ are the roots of $J \mid \Upsilon_{4}$.

(iv) If the positive solution of (7) is $\left\{\Omega_{n}\right\}_{n=-1}^{\infty}$, s.t. (87) along with the following relation holds:

$$
\begin{aligned}
& \bar{x} \in\left[L_{13}, U_{13}\right], \\
& \bar{y} \in\left[L_{14}, U_{14}\right], \\
& \bar{z} \in\left[L_{15}, U_{15}\right],
\end{aligned}
$$

then the error vector, which is depicted in (89), satisfies the following relations:

$$
\begin{array}{r}
\lim _{n \longrightarrow \infty}\left(\left\|\theta_{n}\right\|\right)^{1 / n}=\left|\lambda_{1, \ldots, 6} J\right| \Upsilon_{5} \mid, \\
\lim _{n \longrightarrow \infty} \frac{\left\|\theta_{n+1}\right\|}{\left\|\theta_{n}\right\|}=\left|\lambda_{1, \ldots, 6} J\right| \Upsilon_{5} \mid,
\end{array}
$$

where $\left|\lambda_{1, \ldots, 6} J\right| \Upsilon_{5} \mid$ are the roots of $J \mid \Upsilon_{5}$.

(v) If the positive solution of (8) is $\left\{\Omega_{n}\right\}_{n=-1}^{\infty}$, s.t. (87) along with the following relation holds:

$$
\begin{aligned}
& \bar{x} \in\left[L_{16}, U_{16}\right], \\
& \bar{y} \in\left[L_{17}, U_{17}\right], \\
& \bar{z} \in\left[L_{18}, U_{18}\right],
\end{aligned}
$$

then the error vector, which is depicted in (89), satisfies the following relations:

$$
\begin{gathered}
\lim _{n \longrightarrow \infty}\left(\left\|\theta_{n}\right\|\right)^{1 / n}=\left|\lambda_{1, \ldots, 6} J\right| \Upsilon_{6} \mid, \\
\lim _{n \longrightarrow \infty} \frac{\left\|\theta_{n+1}\right\|}{\left\|\theta_{n}\right\|}=\left|\lambda_{1, \ldots, 6} J\right| \Upsilon_{6} \mid,
\end{gathered}
$$

where $\left|\lambda_{1, \ldots, 6} J\right| \Upsilon_{6} \mid$ are the roots of $J \mid \Upsilon_{6}$.

Proof. It is similar to Theorem 7, and hence its proof is omitted.

\section{Discussion and Simulations}

In the reported work, we have explored the global dynamics of $(2,3)$-type exponential systems of difference equations. We have investigated that $\left\{\Omega_{n}\right\}_{n=-1}^{\infty}$ of systems (3) to (8) is bounded and persists, and the corresponding invariant rectangles, respectively, are $\left[L_{1}, U_{1}\right] \times\left[L_{2}, U_{2}\right] \times\left[L_{3}, U_{3}\right]$, $\left[L_{4}, U_{4}\right] \times\left[L_{5}, U_{5}\right] \times\left[L_{6}, U_{6}\right],\left[L_{7}, U_{7}\right] \times\left[L_{8}, U_{8}\right] \times\left[L_{9}, U_{9}\right]$, $\left[L_{10}, U_{10}\right] \times\left[L_{11}, U_{11}\right] \times\left[L_{112}, U_{12}\right], \quad\left[L_{13}, U_{13}\right] \times\left[L_{14}, U_{14}\right]$ $\times\left[L_{15}, U_{15}\right]$, and $\left[L_{16}, U_{16}\right] \times\left[L_{17}, U_{17}\right] \times\left[L_{18}, U_{18}\right]$. Further, we have explored the existence and uniqueness of the positive equilibrium and the global and local dynamics of systems (3)-(8). We have also investigated the rate of convergence of the positive solution of systems (3)-(8). Finally, some numerical examples are provided to support the theoretical results. For instance, if $\alpha_{i}(i=10, \ldots, 18)$, respectively, are $13,24,319,12,0.1,0.2,1.5,0.4$, and 0.002 , then from Figures 1(a)-1(c), the positive fixed point $(0.06304125281075143,0.567941569702224,0.4220224516$ $533974)$ of (3) is stable and its corresponding attractor is shown in Figure $1(\mathrm{~s})$. Now, if $\alpha_{i}(i=19, \ldots, 27)$, respectively, are $19,14,9,112,0.1,0.2,15,14$, and 2 , then from Figures 1(d)-1(f), the positive equilibrium point $(0.6122$ $355979161732, \quad 0.996719892698477,0.8425637558539959)$ of (4) is stable and its corresponding attractor is shown in Figure 1(t). For (5), if $\alpha_{i}(i=28, \ldots, 36)$, respectively, are 9, $0.4,9,12,0.1,0.2,15,2$, and 15, then from Figures $1(\mathrm{~g})-1(\mathrm{i})$, its unique positive equilibrium point (0.277465951, $0.924713,0.88573)$ is stable and its corresponding attractor is shown in Figure $1(\mathrm{u})$. For (6), if $\alpha_{i}(i=37, \ldots, 45)$, 


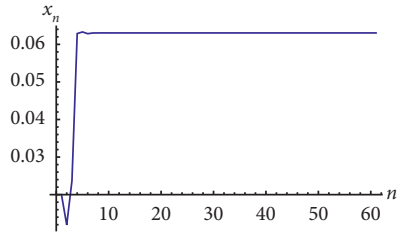

(a)

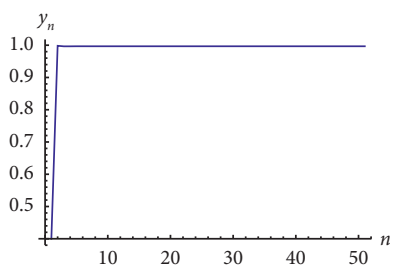

(e)

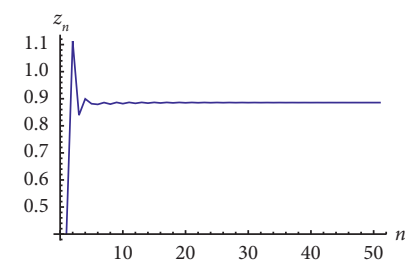

(i)

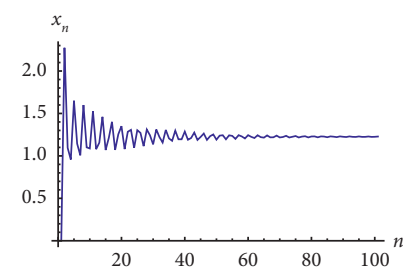

(m)

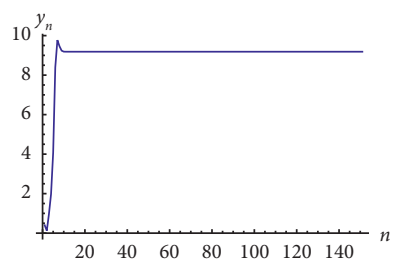

(q)

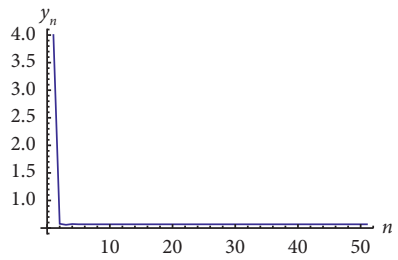

(b)

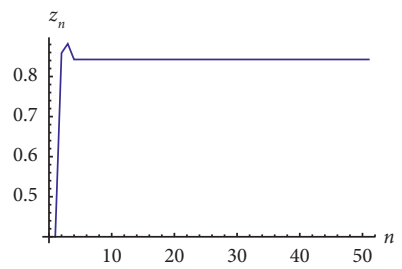

(f)

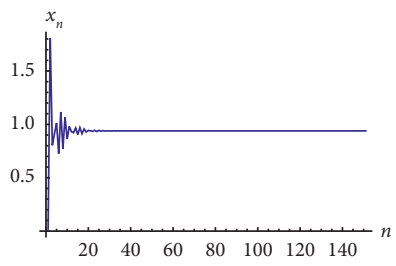

(j)

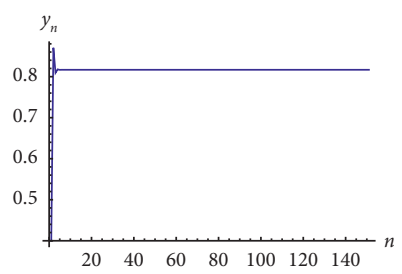

(n)

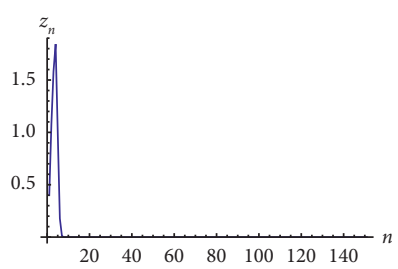

(r)
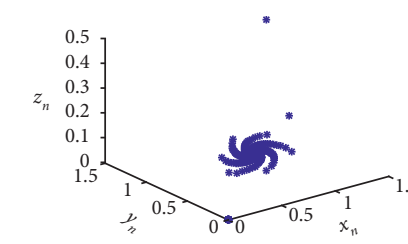

(v)

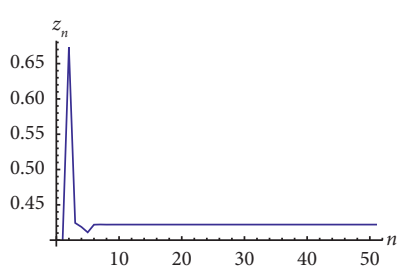

(c)

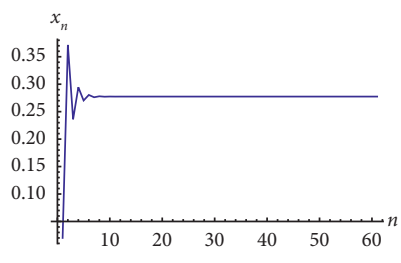

(g)

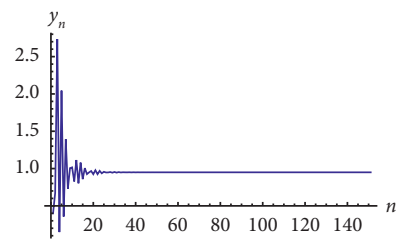

(k)

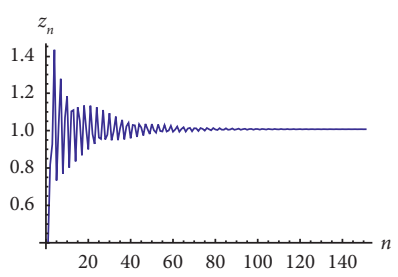

(o)

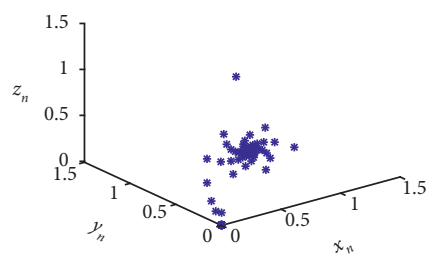

(s)

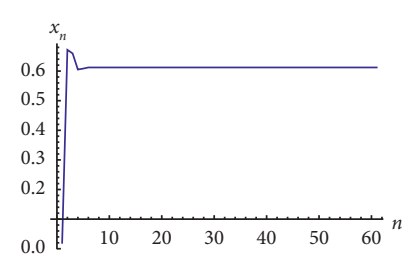

(d)

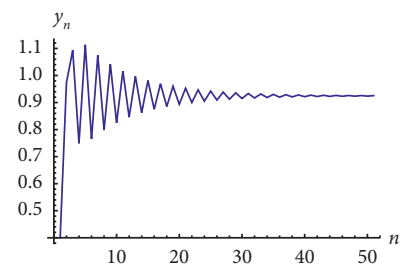

(h)

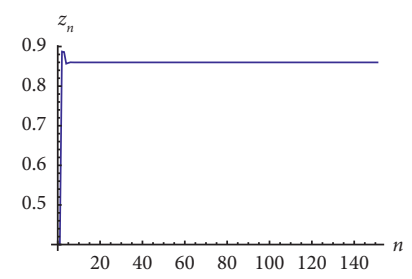

(l)

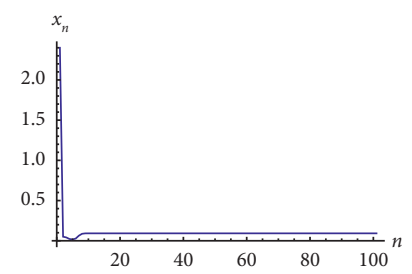

(p)

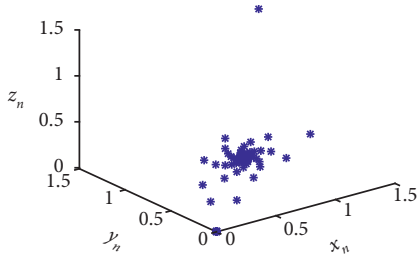

(t)

(u)

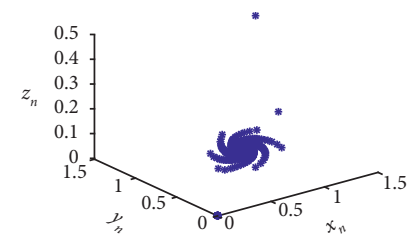

(w)

Figure 1: Trajectories for (3) to (8) with initial values $x_{s}, y_{s}, z_{s}(s=-1,0)$, respectively, being $0.7,2.4,0.9,0.4,1.9$, and 0.4 .

respectively, are $29,14,9,12,0.1,0.2,15,14$, and 2 , then from Figures 1(j)-1(1), its unique positive equilibrium point $(0.9391896591799592,0.9495483631730844,0.8598773120$ 562713 ) is stable and its corresponding attractor is shown in Figure 1(v). For (7), if $\alpha_{i}(i=46, \ldots, 54)$, respectively, are 29, $14,1.9,12,0.1,1.2,15,14$, and 2 , then from Figures 1(m)$1(\mathrm{o})$, its unique positive equilibrium point (1.22564,
$0.8167047,1.007332)$ is stable and its corresponding attractor is shown in Figure $1(\mathrm{v})$. Finally, if $\alpha_{i}(i=55$, $\ldots, 63)$, respectively, are $9,4,129,12,0.1,25,14,14$, and 2 , then from Figures $1(\mathrm{p})-1(\mathrm{r})$, the unique positive equilibrium point $(0.09228515208223682,9.184938317317343$, $0.0000975782517529335)$ of system (8) is stable and its corresponding attractor is shown in Figure 1(w). For more 
results on dynamical properties of difference equations, we refer the reader to $[7,8]$ and the references cited therein.

\section{Data Availability}

All the data utilized in this article have been included and the sources from where they were adopted are cited accordingly.

\section{Conflicts of Interest}

The authors declare that they have no conflicts of interest regarding the publication of this paper.

\section{Acknowledgments}

A. Q. Khan and H. M. Arshad research was partially supported by the Higher Education Commission of Pakistan, while the research of B. A. Younis was funded by a Deanship of Scientific Research in King Khalid University, under grant number GRP-326-40.

\section{References}

[1] I. Ozturk, F. Bozkurt, and S. Ozen, "On the difference equation $x_{n+1}=\left(\alpha_{10}+\alpha_{11} e^{-x_{n}}\right) /\left(\alpha_{12}+x_{n-1}\right)$." Applied Mathematics and Computation, vol. 181, no. 2, pp. 1387-1393, 2006.

[2] F. Bozkurt, "Stability analysis of a nonlinear difference equation," International Journal of Modern Nonlinear Theory and Application, vol. 2, no. 1, pp. 1-6, 2013.

[3] M. Garić-Demirović and M. R. S. Kulenović, "Dynamics of an anti-competitive two dimensional rational system of difference equations," Sarajevo Journal of Mathematics, vol. 7, no. 19, pp. 39-56, 2014.

[4] A. Q. Khan and M. N. Qureshi, "Behavior of an exponential system of difference equations," Discrete Dynamics in Nature and Society, vol. 2014, Article ID 607281, 9 pages, 2014.

[5] A. Q. Khan and M. N. Qureshi, "Qualitative behavior of two systems of higher-order difference equations," Mathematical Methods in the Applied Sciences, vol. 39, no. 11, pp. 3058-3074, 2016.

[6] M. Pituk, "More on poincaré's and perron's theorems for difference equations," Journal of Difference Equations and Applications, vol. 8, no. 3, pp. 201-216, 2002.

[7] T. F. Ibrahim, "Bifurcation and periodically semicycles for fractional difference equation of fifth order," Journal of Nonlinear Sciences and Applications, vol. 11, no. 3, pp. 375-382, 2018.

[8] T. F. Ibrahim, "Behavior of two and three-dimensional systems of difference equations in modelling competitive populations," Dynamics of Continuous, Discrete and Impulsive Systems, Series A: Mathematical Analysis, vol. 24, no. 6, pp. 395-418, 2017. 